\title{
Early and late time VLT spectroscopy of SN 2001el - progenitor constraints for a type la supernova ${ }^{\star}$
}

\author{
S. Mattila ${ }^{1}$, P. Lundqvist ${ }^{1}$, J. Sollerman ${ }^{1}$, C. Kozma ${ }^{1}$, E. Baron ${ }^{2}$, C. Fransson ${ }^{1}$, B. Leibundgut ${ }^{3}$, and K. Nomoto ${ }^{4}$ \\ 1 Stockholm Observatory, AlbaNova, Department of Astronomy, 10691 Stockholm, Sweden \\ e-mail: seppo@astro.su.se \\ 2 Department of Physics and Astronomy, University of Oklahoma, 440 West Brooks Street, Norman, OK 73019, USA \\ 3 European Southern Observatory, Karl-Schwarzschild-Strasse 2, 85748 Garching bei München, Germany \\ 4 Department of Astronomy and Research Center for the Early Universe, University of Tokyo, Bunkyo-ku, Tokyo, Japan
}

Received 20 January 2005 / Accepted 26 September 2005

\section{ABSTRACT}

We present early time high-resolution (VLT/UVES) and late time low-resolution (VLT/FORS) optical spectra of the normal type Ia supernova, SN 2001el. The high-resolution spectra were obtained 9 and 2 days before ( $B$-band) maximum light. This was in order to allow the detection of narrow hydrogen and/or helium emission lines from the circumstellar medium of the supernova. No such lines were detected in our data. We therefore use these spectra together with photoionisation models to derive upper limits of $9 \times 10^{-6} M_{\odot} \mathrm{yr}^{-1} \mathrm{and}_{5} \times 10^{-5} M_{\odot} \mathrm{yr}^{-1} \mathrm{for}_{\mathrm{the}}$ mass loss rate from the progenitor system of SN 2001el assuming velocities of $10 \mathrm{~km} \mathrm{~s}^{-1}$ and $50 \mathrm{~km} \mathrm{~s}^{-1}$, respectively, for a wind extending to outside at least a few $\times 10^{15} \mathrm{~cm}$ away from the supernova explosion site. So far, these are the best $\mathrm{H} \alpha$ based upper limits obtained for a type Ia supernova, and exclude a symbiotic star in the upper mass loss rate regime (so called Mira type stars) from being the progenitor of SN 2001el. The low-resolution spectrum was obtained in the nebular phase of the supernova, 4400 days after the maximum light, to search for any hydrogen rich gas originating from the supernova progenitor system. However, we see no signs of Balmer lines in our spectrum. Therefore, we model the late time spectra to derive an upper limit of $\sim 0.03 M_{\odot}$ for solar abundance material present at velocities lower than $1000 \mathrm{~km} \mathrm{~s}^{-1}$ within the supernova explosion site. According to numerical simulations of Marietta et al. (2000) this is less than the expected mass lost by a subgiant, red giant or a main-sequence secondary star at a small binary separation as a result of the $\mathrm{SN}$ explosion. Our data therefore exclude these scenarios as the progenitor of SN 2001el. Finally, we discuss the origin of high velocity Ca II lines previously observed in a few type Ia supernovae before the maximum light. We see both the Ca II IR triplet and the H\&K lines in our earliest ( -9 days) spectrum at a very high velocity of up to $\sim 34000 \mathrm{~km} \mathrm{~s}^{-1}$. The spectrum also shows a flat-bottomed Si II "6150 ̊” feature similar to the one previously observed in SN $1990 \mathrm{~N}$ (Leibundgut et al. 1991, ApJ, 371, L23) at 14 days before maximum light. We compare these spectral features in SN 2001el to those observed in SN 1984A and SN 1990N at even higher velocities.

Key words. supernovae: general - supernovae: individual: SN 2001el - supernovae: individual: SN 1990N, SN 1984A circumstellar matter - techniques: spectroscopic

\section{Introduction}

While the progenitor stars for a few core-collapse supernovae (SNe), viz. SN 1987A (White \& Malin 1987; Walborn et al. 1989), SN 1993J (Aldering et al. 1994; Maund et al. 2004) and SN 2003gd (Smartt et al. 2004), have been directly detected in pre-explosion images, and a general good picture has emerged from stellar evolutionary modelling (Smartt et al. 2003; Eldridge \& Tout 2004), similar constraints are still missing for the progenitors of thermonuclear (type Ia) supernovae (henceforth SNe Ia). SNe Ia are believed to arise from the thermonuclear explosion of a carbon oxygen white dwarf

* Based on observations collected at the European Southern Observatory, Paranal, Chile (ESO Programmes 67.D-0227 and 69.D-0193). Included in the "The Physics of Type Ia SNe" EC Programme (HPRN-CT-2002-00303; PI: W. Hillebrandt).
(CO WD) in a binary system when its degenerate mass becomes close to the Chandrasekhar limit. Models predict that the companion star can either be another CO WD or a nondegenerate star. In the double degenerate scenario two lowmass CO WDs merge, whereas in the single degenerate scenario matter is accreted from the companion star until the CO WD reaches its limiting mass for carbon ignition (see Hillebrandt \& Niemeyer 2000 and Nomoto et al. 2000 for discussion and references). In neither scenario would the progenitor system be luminous enough to be directly detectable at extragalactic distances. However, the recently reported (RuizLapuente et al. 2004) likely identification of the subgiant/mainsequence companion star surviving the explosion of Tycho Brahe's supernova (SN 1572) gives support for the single degenerate scenarios. 
In all the single degenerate scenarios hydrogen and/or helium rich gas originating from the companion star could be present in the circumstellar medium (CSM) of the SN. This gas could be in the form of a stellar wind, an accretion disk, a filled Roche-lobe, a common envelope around the progenitor system, or a wind from the accreting WD (Hachisu et al. 1999a,b). This material could be detectable by making use of the $\mathrm{SN}$ as a probe of its own CSM. If the wind is dense enough and ionised by the radiation from the $\mathrm{SN}$ ejecta/wind interaction, narrow emission lines similar to those in narrow-line (type IIn) corecollapse SNe (and SN 1987A at late times) could be visible on top of the SN spectrum (Cumming et al. 1996; Lundqvist et al. 2006). In fact, SN 2002ic was recently observed to show spectral features similar to both SNe Ia and SNe IIn (Hamuy et al. 2003; Baron 2003; Deng et al. 2004; Kotak et al. 2004); it could belong to the so-called type $1.5 \mathrm{SNe}$ (e.g., Chugai et al. 2004; Nomoto et al. 2004) which were predicted by Iben \& Renzini (1983). The SN Ia explosion itself is also expected to cause a loss of material from a close, non-merging companion (Fryxell \& Arnett 1981; Taam \& Fryxell 1984; Livne et al. 1992; Marietta et al. 2000). This material is likely to be hydrogen rich, and could reveal itself when the ejecta have become transparent.

In addition, Ca II IR triplet ( $8498 \AA, 8542 \AA, 8662 \AA)$ and H\&K (3968 ̊, $3934 \AA)$ lines have recently been observed at very high velocities compared to the $\mathrm{SN}$ photospheric lines in a few SNe Ia: SN 1994D (Hatano et al. 1999), SN 1999ee (Mazzali et al. 2005a), SN 2000cx (Thomas et al. 2004; Branch et al. 2004), SN 2001el (Wang et al. 2003; Kasen et al. 2003), SN 2003du (Gerardy et al. 2004) and SN 2004dt (Wang et al. 2004). While the origin of these high velocity (HV) lines is still unknown, one of the interpretations has been interaction of the SN ejecta with CSM material originating from the companion star (e.g., Gerardy et al. 2004).

To shed light on the origin of SNe Ia we have been conducting a Target of Opportunity (ToO) programme on the European Southern Observatory (ESO) Very Large Telescope (VLT). We have observed two SNe Ia so far, SNe 2000cx and 2001el with the VLT (for preliminary analyses see Lundqvist et al. 2003, 2005). The observations of SN 2000cx, and their modelling, are described in Lundqvist et al. (2006). Here we report our observations of SN 2001el.

Supernova 2001el was discovered on September 17.1 UT (Monard 2001) about 14" west and 20" north of the nucleus of an edge-on spiral galaxy NGC 1448 . This nearby $\left(V_{\text {rec }}=\right.$ $1164 \mathrm{~km} \mathrm{~s}^{-1}$; de Vaucouleur et al. 1991) spiral has hosted also two type II SNe (SN 1983S and SN 2003hn) during the last 20 years. The magnitude of SN 2001el at the time of the discovery was about 14.5. It was classified as a SN Ia by us on September 21.3 UT (Sollerman et al. 2001). SN 2001el was observed to show a polarised spectrum before the maximum light (Wang et al. 2003) indicating some asymmetric structure during these initial phases of expansion. Later on, SN 2001el has been well monitored both photometrically and spectroscopically, and has been shown to be a normal SN Ia with $\Delta m_{15}(B)=1.13$ (Krisciunas et al. 2003).

In Sect. 2 the VLT observations and data reductions are described. In Sect. 3, we describe how we searched for
CSM lines from the early time high-resolution and late time low-resolution spectra of SN 2001el. No CSM lines were detected and thus upper limits were derived for the line fluxes. In Sect. 4, we use these flux limits together with models to estimate upper limits for the amount of hydrogen rich material lost from the companion star either via a stellar wind or as a result of the SN explosion. In Sect. 5, we compare the profiles of the high velocity $\mathrm{Ca}$ II IR triplet and $\mathrm{H} \& \mathrm{~K}$ lines as well as the flat-bottomed Si II " $6150 \AA$ " feature in our early time spectrum to those observed in SNe 1984A and $1990 \mathrm{~N}$ at a similar early epoch. Finally, in Sect. 6 we discuss our results and present the summary.

\section{VLT observations and data reductions}

\subsection{High-resolution UVES spectroscopy before the maximum light}

Two days after the discovery IAU circular (Monard 2001) on September 21.3 (UT), SN 2001el, still without a spectroscopic classification, was observed with the Ultraviolet and Visual Echelle Spectrograph (UVES) on the VLT. All the observations were obtained in service mode. We obtained two 2400 second exposures in each of two different dichroic settings, DIC1 390+564 and DIC2 437+860 (Table 1). These settings together give a complete wavelength coverage between $3260 \AA$ and $10600 \AA$. The night was clear and the seeing during the observations was around $0 . ' 8$. The spectrophotometric standard star LTT 1020 (Hamuy et al. 1994) was also observed in both setups, after the supernova observations. All spectra were quickly reduced using the UVES pipeline. This allowed a classification of the supernova as a type Ia, well before the maximum light (Sollerman et al. 2001).

The SN was observed again, 7 days later, on September 28.3 (UT) to enable monitoring of any weak CSM lines potentially detectable from this promising early SN Ia event. Three exposures of 3000 seconds each were obtained using the dichroic setting DIC1 $390+564$, which covers the wavelength ranges $3260-4450 \AA$ and $4580-6680 \AA$. The night was clear and the seeing was around 1". 1 during the observations. The spectrophotometric standard star HR 1996 (Tinney \& Reid 1998) was observed immediately after the supernova observations. The 0.8 slit was used for all the observations on both epochs yielding a spectral resolution of $R \sim 50000$ $\left(\sim 6 \mathrm{~km} \mathrm{~s}^{-1}\right)$ for the region around $\mathrm{H} \alpha$. Our UVES observations of SN 2001el were thus obtained 8.7 and 1.7 days before the supernova $B$-band maximum light, which occurred on September 30.0 (UT) (Krisciunas et al. 2003).

\subsection{UVES data reductions}

The spectral orders covering the lines of interest for this study were carefully reduced using a combination of tasks from the UVES pipeline ${ }^{1}$ (Ballester et al. 2003) (version 2.0) and IRAF. All the 2D data were interactively reduced using the UVES-pipeline task REDUCE/SPAT and calibration frames

\footnotetext{
${ }^{1}$ http://www. eso.org/observing/dfo/quality/
} 
Table 1. Log of VLT observations of SN 2001el.

\begin{tabular}{|c|c|c|c|c|c|c|c|c|}
\hline Instrument & Date (UT) & $\begin{array}{l}\text { JD } \\
(2452000+)\end{array}$ & $\begin{array}{l}\text { Epoch } \\
\text { (days) }\end{array}$ & $\begin{array}{l}\text { Exp. } \\
(\mathrm{sec})\end{array}$ & a.m. $^{a}$ & $\begin{array}{l}\text { Seeing }{ }^{b} \\
(\operatorname{arcsec})\end{array}$ & set-up & $\begin{array}{l}\text { Slit width } \\
\text { (arcsec) }\end{array}$ \\
\hline UVES & 2001 Sep. 21.22 & 173.72 & -8.7 & 2400 & 1.30 & 0.8 & $390+564^{c}$ & 0.8 \\
\hline UVES & 2001 Sep. 21.25 & 173.75 & -8.7 & 2400 & 1.19 & 0.8 & $390+564$ & 0.8 \\
\hline UVES & 2001 Sep. 21.28 & 173.78 & -8.7 & 2400 & 1.12 & 0.7 & $437+860^{d}$ & 0.8 \\
\hline UVES & 2001 Sep. 21.31 & 173.81 & -8.7 & 2400 & 1.08 & 0.9 & $437+860$ & 0.8 \\
\hline UVES & 2001 Sep. 28.22 & 180.72 & -1.7 & 3000 & 1.20 & 1.1 & $390+564$ & 0.8 \\
\hline UVES & 2001 Sep. 28.26 & 180.76 & -1.7 & 3000 & 1.11 & 1.1 & $390+564$ & 0.8 \\
\hline UVES & 2001 Sep. 28.29 & 180.79 & -1.7 & 3000 & 1.07 & 1.1 & $390+564$ & 0.8 \\
\hline FORS1 & 2002 Nov. 02.15 & 580.65 & +398 & 3000 & 1.15 & 1.2 & GRIS_300V & 1.3 \\
\hline
\end{tabular}

a The effective airmass of the observation.

${ }^{b}$ Seeing from the DIMM-monitor.

c Setting 390+564 covers wavelength ranges 3260-4450 A, 4580-6680 A.

${ }^{d}$ Setting 437+860 covers wavelength range 3730-4990 ̊, 6600-10 $600 \AA$.

obtained during daytime operation. This included bias subtraction, flat fielding the data in pixel space, automatically determining the Echelle order positions and slopes, and subtraction of the inter-order (median) background. The pipeline performed wavelength calibration for the $2 \mathrm{D}$ data by automatically identifying and fitting a large number $(\sim 1300$ per set-up) of ThAr arc lines. For the wavelength calibration a single dispersion solution, obtained for the center coordinate of the object, is used for each pixel in the spatial direction. The pipeline then produces separate 2D frames for each Echelle order with constant wavelength binning on the $x$-axis, and the spatial extent of the data on the $y$-axis.

An accurate correction for the background emission from the supernova host galaxy is vital when searching for traces of weak emission or absorption lines from the supernova CSM (see also Della Valle et al. 1996). In fact, the widths and luminosities of emission lines expected from the CSM of a SN Ia are similar to the ones observed from ordinary $\mathrm{H}$ II regions. The luminosity time evolution of such circumstellar emission lines can also be slow enough (in a timespan of a few days) to result in confusion with the constant emission from a nearby H II region. We performed the background subtraction outside the UVES pipeline using the IRAF BACKGROUND task interactively. We found that the dispersion solution obtained for the center coordinate of the object was valid over the slit length (8-12") enabling accurate subtraction of the sky and host galaxy background emission from the wavelength calibrated frames.

The UVES pipeline uses a Gaussian approximation for the cross-order light profile during the optimal extraction. This is not valid when the signal to noise ratio $(\mathrm{S} / \mathrm{N})$ is high enough (Piskunov \& Valenti 2002; Ballester et al. 2003). In fact, we found that the standard optimal extraction routines included, e.g., in the UVES pipeline and IRAF did not give satisfactory results for our Echelle spectra with high S/N. Therefore, in this study we decided to use simple average extraction for the SN and standard star spectra, and performed the cosmic ray rejection when combining the 1D spectra.

To correct for the telluric absorption lines and any residual instrumental features left after the flat fielding, the supernova spectra were divided by the spectrum of the standard star observed during the same night as the supernova with the same instrument settings and scaled to match the effective airmass of the science observation. Both the standard stars observed, LTT 1020 and HR 1996, show broad $\mathrm{H} \alpha$ absorption lines with their wings extending close to the region of interest when searching for the circumstellar $\mathrm{H} \alpha$ line from the supernova spectrum. We therefore normalised the wavelength regions of interest in the standard star spectra with a low order polynomial fit before the division of the supernova data. The LTT 1020 spectrum was also smoothed before the division in order to conserve the $\mathrm{S} / \mathrm{N}$ of the science data. The corrected science exposures were then normalised by a low order polynomial fit, and average combined together (see Figs. 3 and 4). For this we used weighting by the inverse squares of the corresponding pixel to pixel noise levels. Any deviant pixels or cosmic rays (above $5 \sigma$ level) were rejected and replaced by the average of the two unaffected spectra or in the case of only two available spectra by a fit to the continuum. The individual orders covering the wavelengths around the $\mathrm{Ca}$ II (H\&K and the IR triplet) and the Si II " $6150 \AA$ ^" lines were carefully merged together in IRAF (see Fig. 8).

The absolute flux calibration was performed for each wavelength region of interest separately. For this we used the wellsampled $V$-band light curve of SN 2001el from Krisciunas et al. (2003) and a low-resolution spectrum from Wang et al. (2003). This spectrum was observed on September 26.3 (UT), i.e., 4.0 days before the maximum light. To estimate the uncertainties due to the spectral evolution between the epochs of our UVES spectra and the spectrum of Wang et al. (2003) we used low-resolution spectra of SN 2002bo (Benetti et al. 2004) from 9.0, 4.0 and 2.0 days before the maximum light. These are both normal $\mathrm{SNe}$ Ia with exactly the same $\Delta m_{15}$ values (Krisciunas et al. 2003; Benetti et al. 2004). The low-resolution spectra were scaled in flux to match the observed $V$-band photometry at the dates of our UVES observations. From this comparison we estimate that our absolute flux calibration is accurate to better than $\pm 25 \%$.

The reduction procedure outlined above was designed to mainly study limited wavelength regions around narrow, and presumably weak line features. To obtain an overall spectrum of the SN we do not need such a high accuracy. For the first epoch ( -8.7 day) overall spectrum we therefore simply 
used the standard UVES pipeline task REDUCE/UVES. Here the individual orders were automatically extracted and merged together by the pipeline. To enable successful automatic merging, simple average extraction was used, and the individual orders were flat fielded in 1D. The relative flux calibration was carried out in IRAF separately for the spectra corresponding to each spectrograph set-up (see Table 1). For this we used the standard star (LTT 1020) spectra observed together with the SN. To remove the large number of cosmic rays we median filtered each individual spectrum using a filter window of 50 pixels $\left(\sim 50 \mathrm{~km} \mathrm{~s}^{-1}\right)$. The overlapping spectra were average combined together, and rebinned to yield a pixel scale of $\sim 50 \mathrm{~km} \mathrm{~s}^{-1}$ pixel $^{-1}$. The resulting spectrum is shown in Fig. 1 where the levels of the wavelength regions covered by the different set-ups have been adjusted by eye to match the overall shape of the -4 day low-resolution spectrum of Wang et al. (2003). The overall level of the spectrum has been scaled in flux to match the observed $V$-band photometry of the SN.

\subsection{Low-resolution FORS spectroscopy in the nebular phase}

We have also obtained a very late low-resolution optical spectrum of SN 2001el. On November 2.2 (UT) 2002, 398 days past maximum light, we used FORS1 on the VLT to obtain a $3000 \mathrm{~s}$ exposure (see Table 1 for details). We used the $300 \mathrm{~V}$ grism together with order-sorting filter GG375 and a 1'. 3 wide slit yielding a spectral resolution of $R \sim 440\left(\sim 700 \mathrm{~km} \mathrm{~s}^{-1}\right)$ for the observation.

The spectrum (see Fig. 6) was reduced in a standard way in IRAF, including bias subtraction, flat fielding, and wavelength calibration using spectra of a helium-argon lamp. Flux calibration was done relative to the spectrophotometric standard star LTT 2415 (Hamuy et al. 1994). We have also compared the synthetic $V$ band magnitude obtained from this spectrum to late time light curves of SNe Ia (e.g., Sollerman et al. 2004, their Fig. 11) and conclude that the absolute fluxing of the spectrum is accurate to $\pm 20 \%$.

\section{Searching for traces of circumstellar lines}

\subsection{The expected wavelength range for the CSM lines}

Superposed on the supernova spectra (Fig. 1) we also detect interstellar Ca II H\&K absorption both from the Galaxy and from NGC 1448 which hosted the supernova. The Ca II $\mathrm{H} \& \mathrm{~K}$ absorption lines arising within the host galaxy are plotted in Fig. 2 as observed on Sep. 28. Several absorption components are present in the spectrum (see detailed analysis in Sollerman et al. 2005). The overall range of velocities for the absorbing gas along the line-of-sight to the supernova is $V_{\text {abs }} \sim 1130-1230 \mathrm{~km} \mathrm{~s}^{-1}$. The recession velocity of the $\mathrm{H}$ II regions close to the supernova included in the UVES slit can be used as another indicator of the supernova recession velocity. We measured $V_{\mathrm{H} \text { II }}=(1186 \pm 45) \mathrm{km} \mathrm{s}^{-1}$ for the $\mathrm{H} \alpha$ emitting gas next to the SN position in the UVES spectrum at Sep. 28. As NGC 1448 is oriented almost edge on $\left(i=88^{\circ}\right)$ the galaxy rotation may result in a substantial velocity for the supernova w.r.t. to the host galaxy recession velocity. A rotation velocity of $V_{\text {rot }} \sim 193 \mathrm{~km} \mathrm{~s}^{-1}$ has been measured for NGC 1448 (Mathewson \& Ford 1996). We therefore carry-out the search for the narrow circumstellar emission lines at the range of velocities $V_{\text {rec }}=(1180 \pm 190) \mathrm{km} \mathrm{s}^{-1}$. This is close to the recession velocity of NGC 1448 of $1164 \pm 5 \mathrm{~km} \mathrm{~s}^{-1}$ compiled by de Vaucouleur et al. (1991).

\subsection{Early time spectra}

The overall UVES spectrum obtained on Sep. 21.3 (UT), i.e., 8.7 days before the $B$-band maximum light is shown in Fig. 1 . The spectrum of SN $1990 \mathrm{~N}$ observed at a very early epoch of $\sim 14$ days before the $B$-band maximum light (Leibundgut et al. 1991 ) is also shown for comparison. Although SN $1990 \mathrm{~N}$ was at a much earlier phase than SN 2001el at the time of the observation these spectra appear remarkably similar. Both $\mathrm{SNe}$ show strong Ca II IR triplet and H\&K lines at similar high velocities. The characteristic Si II "6150 ̊” absorption appears flat-bottomed in both the spectra. To our knowledge such flatbottomed Si II lines have not been seen in any other SNe Ia. The origin of these lines will be discussed in Sect. 5 .

The strongest lines expected to form in a hydrogen or helium-rich companion wind, and which are covered by our spectral range, i.e., $\mathrm{H} \alpha$, or He I $\lambda 5876$ and He II $\lambda 4686$ (cf. Lundqvist et al. 2006), were searched for in the UVES spectra at the two epochs of observation (see Figs. 3 and 4). For the predicted temperatures of $\sim 20000-40000 \mathrm{~K}$ (Lundqvist et al. 2006) thermal broadening dominates the line widths for wind velocities up to $\sim 40 \mathrm{~km} \mathrm{~s}^{-1}$ for hydrogen and $\sim 20 \mathrm{~km} \mathrm{~s}^{-1}$ for helium. Here we will assume an average temperature of $28000 \mathrm{~K}$ (for details see Lundqvist et al. 2006) and a wind velocity, $V_{\text {wind }}$, between $10 \mathrm{~km} \mathrm{~s}^{-1}$ and $50 \mathrm{~km} \mathrm{~s}^{-1}$, and therefore adopt observed line widths of $37-62 \mathrm{~km} \mathrm{~s}^{-1}$ and $21-53 \mathrm{~km} \mathrm{~s}^{-1}$ for hydrogen and helium lines, respectively.

No narrow lines with the expected line widths were detected in our spectra and therefore only upper limits for the emission line fluxes were obtained. To estimate detection limits for the narrow CSM emission lines we used the IRAF FITPROFS task to perform least squares fitting of Gaussian profiles (one at a time) to the normalised and unbinned spectra. The free parameters were the amplitude, the width (sigma) and the wavelength position of the Gaussian. As the spectra were already normalised, we did not fit the background. The $1 \sigma$ width of the Gaussian profile was fixed according to the relevant $F W H M$, and the line center was moved through the spectrum in small $(F W H M / 2)$ steps, i.e., only the amplitude of the profile was kept as a free parameter. To obtain statistically meaningful upper limits lines were fitted to a $\pm 2 \times 190 \mathrm{~km} \mathrm{~s}^{-1}$ region around the most probable location of the CSM line with similar noise characteristics. This procedure yields a list of potential emission and absorption lines through the spectrum. In order to derive reliable flux limits from the data, the histogram distributions of the peak flux densities of the line profiles were inspected. These histograms have centers close to zero flux density and can be fitted with Gaussian profiles (see Fig. 5 (upper panel)) yielding reduced $\chi^{2} \mathrm{~s}$ of around 2-3 as expected 


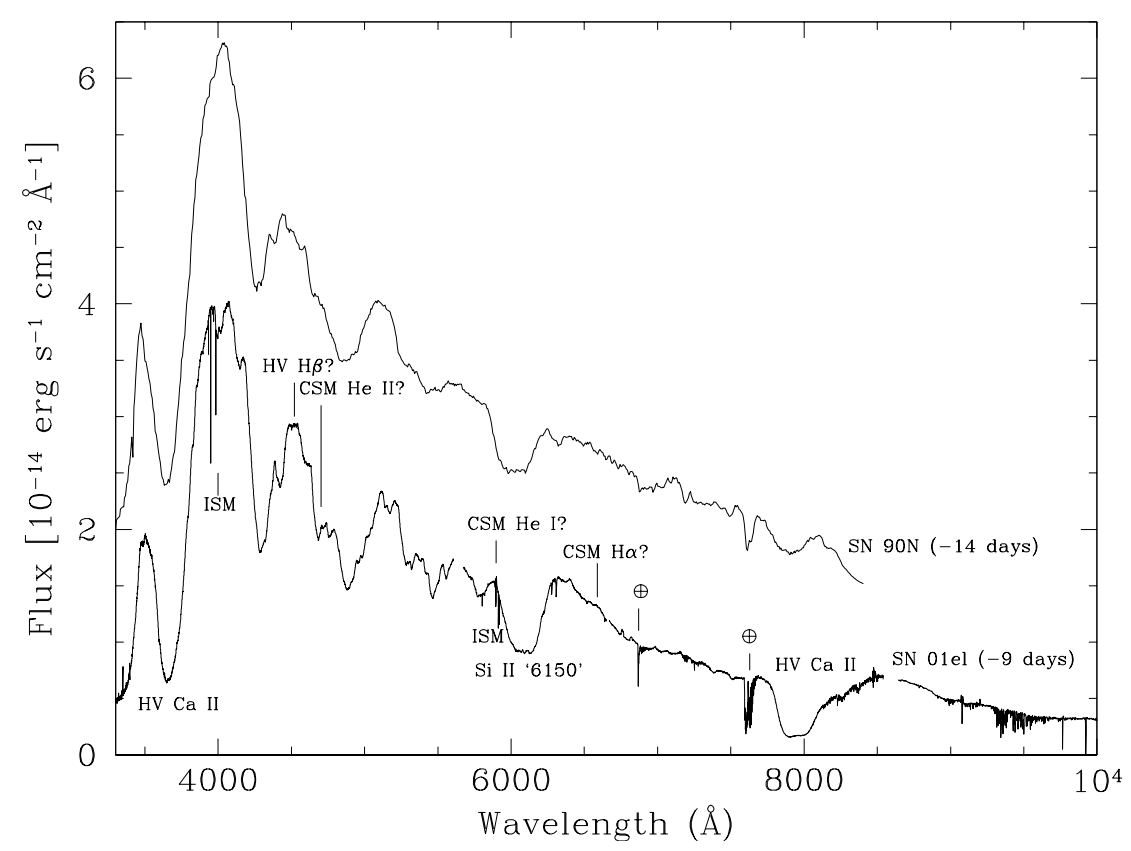

Fig. 1. UVES spectrum of SN 2001el obtained on September 21.3 (UT), i.e., 8.7 days before the $B$-band maximum light. The spectrum has been median filtered and rebinned to have a resolution of about $50 \mathrm{~km} \mathrm{~s}^{-1} \mathrm{pixel}^{-1}$. The lines of interest for this study are indicated. The spectrum of SN 1990N observed (Leibundgut et al. 1991) at 14 days before the $B$-band maximum light is also shown for comparison. This spectrum has been redshifted to match the recession velocity of SN 2001el, scaled by $\times 3.5$, and shifted upward by $1.5 \times 10^{-14} \mathrm{erg} \mathrm{s}^{-1} \mathrm{~cm}^{-2} \AA^{-1}$ for clarity. The Ca II IR triplet and the H\&K lines are present at a very high velocity (HV), and the characteristic Si II "6150 ̊”" absorption shows a similar strongly blueshifted flat-bottomed profile in both SNe. However, we see no signs of narrow circumstellar hydrogen or helium lines nor HV H $\beta$ in the SN 2001el spectrum. The Ca II and Na I ISM lines are also marked in the SN 2001el spectrum (for details see Sollerman et al. 2005). A large number of telluric lines are present, especially at wavelengths longer than $9000 \AA$.

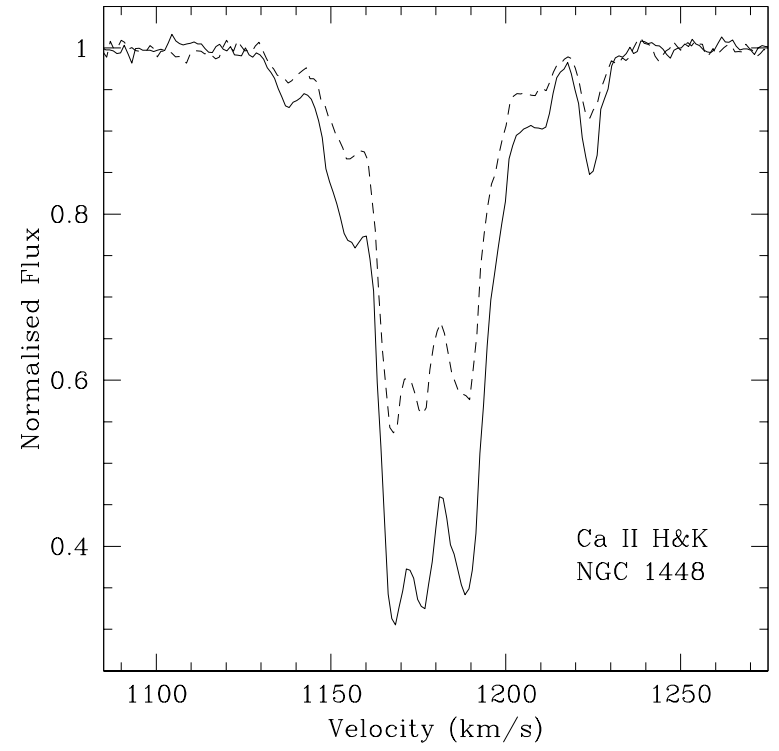

Fig. 2. Ca II H ( $\lambda 3968$, dashed) and K ( $\lambda 3934$, solid) interstellar absorption lines towards SN 2001el within its host galaxy NGC 1448. The velocity scale is heliocentric. See Sollerman et al. (2005) for details about the absorption components.

from pure random noise. The centers $(\mu)$ and $1 \sigma$ widths of these distributions together with the derived $3 \sigma$ emission line upper limits $(f(3 \sigma)=\mu+3 \times \sigma)$ are listed in Table 2. For comparison we also show $3 \sigma$ upper limits derived simply from the measured pixel-to-pixel standard deviations in the spectra rebinned to have a Nyquist sampling, i.e., one pixel equal to $F W H M / 2$ for each expected line width (see above). This simple approach yields $3 \sigma$ limits consistent with the more mathematically robust method described above.

In Fig. 5 (lower panel) we show the wavelength region around the expected position of the $\mathrm{H} \alpha$ line in our first epoch spectrum together with a Gaussian profile with $F W H M=37 \mathrm{~km} \mathrm{~s}^{-1}$ and its peak at the $3 \sigma$ level as obtained using the method described above. For lines of $20-60 \mathrm{~km} \mathrm{~s}^{-1}$ the reduced UVES data with a $0.77 \mathrm{~km} \mathrm{~s}^{-1}$ pixel size are strongly over-sampled. Therefore, a comparison of the upper limits derived above with sufficiently rebinned data is more meaningful. In Fig. 5 (lower panel) we show both the unbinned $\left(0.77 \mathrm{~km} \mathrm{~s}^{-1}\right.$ pixel $\left.^{-1}\right)$ spectrum and a spectrum rebinned to $18 \mathrm{~km} \mathrm{~s}^{-1}$ pixel $^{-1}$. We note that there are no features with fluxes at the level or higher than the estimated $3 \sigma$ level present in the rebinned data at the expected wavelength range of $\mathrm{H} \alpha$. The derived $3 \sigma$ upper limits for the hydrogen and helium lines at the two different epochs are given in Table 3. For the first epoch observation we also give $\mathrm{H} \alpha$ upper limits for higher wind velocities of $100 \mathrm{~km} \mathrm{~s}^{-1}$ and $200 \mathrm{~km} \mathrm{~s}^{-1}$. These limits are simply based on the pixel-to-pixel standard deviations in the rebinned $(1$ pixel $=F W H M / 2)$ spectra between $6570 \AA$ and $6608 \AA$.

\subsection{Late time spectra}

In Fig. 6 we show the observed nebular spectrum of SN 2001el at 398 days after maximum light. The SN Ia spectra at these 


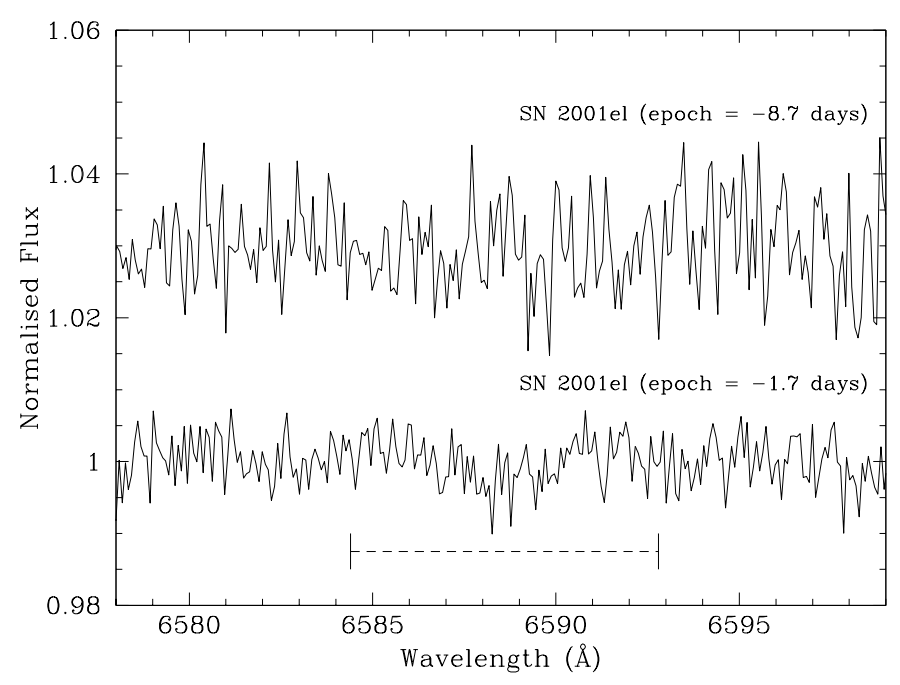

Fig. 3. Normalised and rebinned $\left(\sim 4 \mathrm{~km} \mathrm{~s}^{-1}\right.$ pixel $\left.^{-1}\right)$ UVES spectra in the expected spectral region around $\mathrm{H} \alpha$ for SN 2001el on two epochs, September 21.3 and 28.3 (UT) 2001, i.e., 8.7 and 1.7 days before the SN maximum light, respectively. The expected wavelength range of $\mathrm{H} \alpha$ is marked with a horizontal dashed line, and the upper spectrum has been shifted vertically for clarity. No significant emission or absorption lines are visible.

epochs are dominated by $\mathrm{Fe}$ emission. The features between $4000 \AA$ and $5500 \AA$ are blends of Fe II and Fe III emission, while the features in the range $7000 \AA$ to $7600 \AA$ are mainly due to Fe II and Ni II. By comparing the observations to detailed late time modelling we are able to put a conservative upper limit on the amount of solar abundance material evaporated from a binary companion. Following the simulations of Marietta et al. (2000) we have searched in our spectra for signs of relatively narrow $\left(F W H M \lesssim 1000 \mathrm{~km} \mathrm{~s}^{-1}\right.$ ) $\mathrm{H} \alpha$ emission at the range of wavelengths corresponding to the recession velocity found in Sect. 3.1. No such emission is apparent in the spectrum. Our model calculations are described in Sect. 4.3.

\section{Constraints on the progenitor system of SN 2001el}

\subsection{Extinction and distance of SN 2001el}

In order to estimate the upper limit for the mass loss rate, $\dot{M}$, from the progenitor system of SN 2001el we need to know the upper limits for the line luminosities, i.e., we need to know the extinction and distance towards the supernova. Krisciunas et al. (2003) have measured a colour excess of $E(B-V)=$ $0.253 \pm 0.063$ for SN 2001el (see also Sollerman et al. 2005) using the Lira relation (Phillips et al. 1999). Here we adopt $E(B-V)=0.25 \pm 0.06$, i.e., $A_{\mathrm{V}}=0.78 \pm 0.19$ (assuming $\left.R_{V}=3.1\right)$ for the extinction towards the supernova.

Probably the most accurate method for deriving distances for normal SNe Ia is by making use of their photometry. Krisciunas et al. (2003) observed $m(\mathrm{H})_{14}=13.18 \pm 0.05$ for the $\mathrm{SN}$ at +14 days from the $B$-band maximum light. Meikle (2000) estimated $M(\mathrm{H})_{14}=-18.22 \pm 0.05$ for "IRnormal" SNe Ia. Assuming the Galactic extinction law of Rieke \& Lebofsky (1985) we have an $H$-band extinction of
$A_{\mathrm{H}}=0.14 \pm 0.03$ towards the supernova. This results in a distance of $17.9 \pm 0.7 \mathrm{Mpc}$ for the supernova. We note that Krisciunas et al. (2003) obtained exactly the same distance $(17.9 \pm 0.8 \mathrm{Mpc})$ for $\mathrm{SN} 2001 \mathrm{el}$ using their $B V I$ light curves and the method of Phillips et al. (1999). As the two distance estimates obtained by making use of the supernova photometry are identical we adopt $17.9 \pm 0.8 \mathrm{Mpc}$ for the distance of SN 2001el.

\subsection{Upper limit for the progenitor mass loss rate}

We used our estimated upper limits for the narrow $\mathrm{H} \alpha$ line luminosity together with the photoionisation models from Lundqvist et al. (2006) to obtain upper limits for the mass loss rate from the supernova progenitor system. In these calculations wind velocities of $10 \mathrm{~km} \mathrm{~s}^{-1}$ and $50 \mathrm{~km} \mathrm{~s}^{-1}$ and solar abundances were assumed. The model in Lundqvist et al. (2006) is an updated version of that described in Cumming et al. (1996). We still assume spherical symmetry and powerlaw density distributions of the SN ejecta and the circumstellar gas, where the ejecta profile is assumed to follow $\rho_{\text {ejecta }} \propto r^{-7}$, and the wind has the standard $\rho_{\text {wind }} \propto r^{-2}$ distribution. The shock structure, and blast wave expansion, can then be described by the similarity solutions of Chevalier (1982). The shocked gas produces hard radiation that ionises the unshocked wind and gives rise to narrow circumstellar lines. A difference compared to Cumming et al. (1996) is that we now assume much higher, and therefore more realistic, velocities of the ejecta (see also Sect. 6). The velocity of the fast circumstellar shock is in our present models $V_{\mathrm{s}} \sim 4.45 \times 10^{4} \mathrm{~km} \mathrm{~s}^{-1}$ at day 1 , which is about twice as fast as in Cumming et al. (1996). The use of such a high shock velocity is supported by the recent observations of high velocity Ca II lines in early SN Ia spectra (see Sect. 5 and references therein) with their maximum velocities up to $\sim 35000 \mathrm{~km} \mathrm{~s}^{-1}$, especially since the circumstellar shock can be expected to advance $\sim 1.2-1.3$ times (Chevalier 1982) faster than the fastest unshocked ejecta that gives rise to the Ca II lines. In addition, the maximum velocity of unshocked ejecta decreases with time, so the bluest absorption observed in the ejecta 1-2 weeks before the maximum light (see Table 3) can only give a lower limit to the maximum velocity of unshocked ejecta at even earlier times. A dense wind will therefore be overtaken much faster. Compared to Cumming et al. (1996) we have also calculated the free-free emission from the shocked ejecta more accurately (cf. Lundqvist et al. 2006).

Adopting an extinction of $A_{\mathrm{V}}=0.78 \pm 0.19$, a distance of $D=17.9 \pm 0.8 \mathrm{Mpc}$, and $V_{\text {wind }}=10 \mathrm{~km} \mathrm{~s}^{-1}$ for the SN 2001el progenitor system, we obtain $3 \sigma$ upper limits for the $\mathrm{H} \alpha$ narrow line-luminosity of $L_{\mathrm{H} \alpha}<5.3 \times 10^{36} \mathrm{erg} \mathrm{s}^{-1}$ and $L_{\mathrm{H} \alpha}<6.9 \times 10^{36} \mathrm{erg} \mathrm{s}^{-1}$ for the first ( -8.7 days) and the second ( -1.7 days) epoch, respectively. If a higher wind velocity of $50 \mathrm{~km} \mathrm{~s}^{-1}$ is assumed, the luminosity upper limits are $L_{\mathrm{H} \alpha}<6.9 \times 10^{36} \mathrm{erg} \mathrm{s}^{-1}$ and $L_{\mathrm{H} \alpha}<8.3 \times 10^{36} \mathrm{erg} \mathrm{s}^{-1}$ for the two epochs, respectively. In Fig. 7 these upper limits are plotted together with modelled line luminosities for six different progenitor mass loss rates. Here we have assumed a rise time of $19.5 \pm 0.2$ days for SN 2001el which is typical for normal 

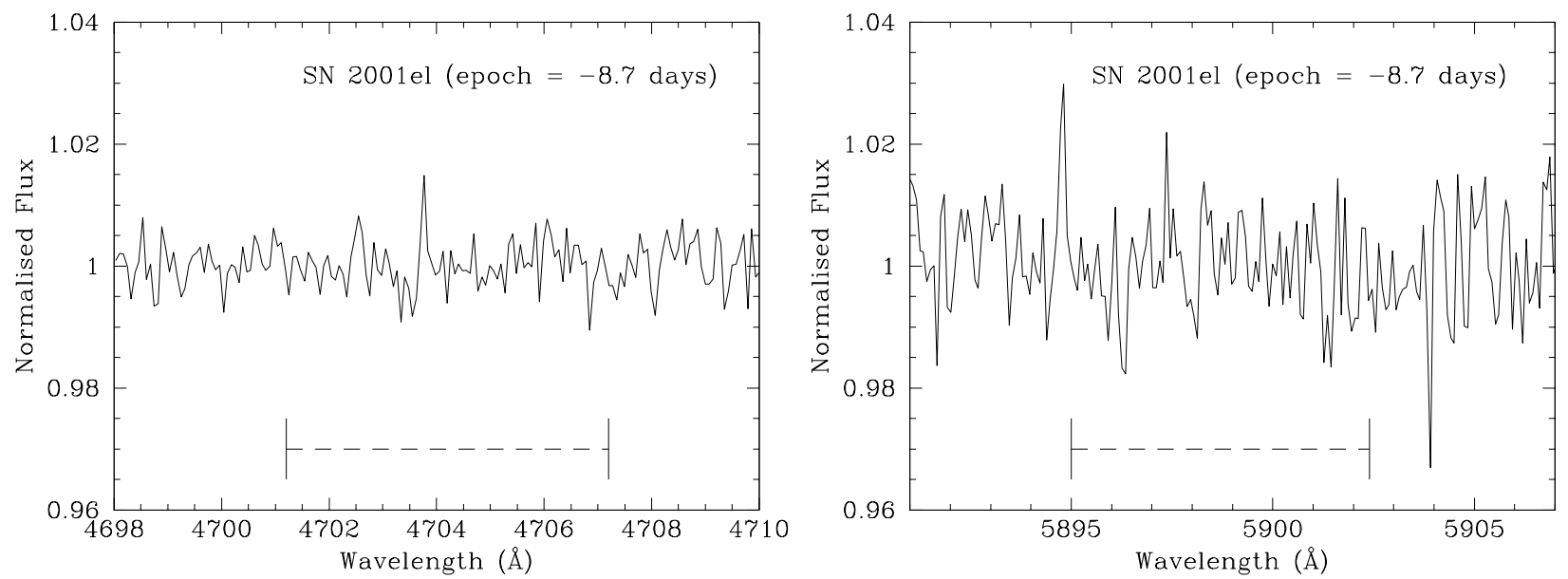

Fig. 4. Normalised and rebinned ( $\sim \mathrm{km} \mathrm{s}^{-1}$ pixel $\left.^{-1}\right)$ UVES spectra in the expected spectral regions around He II (4686 $\left.\mathrm{A}\right)$ and He I (5876 ̊̊) for SN 2001el on September 21.3 (UT) 2001, i.e., 8.7 days before the maximum light. The expected wavelength range of the CSM lines is marked with a horizontal dashed line. No significant emission or absorption lines are visible.
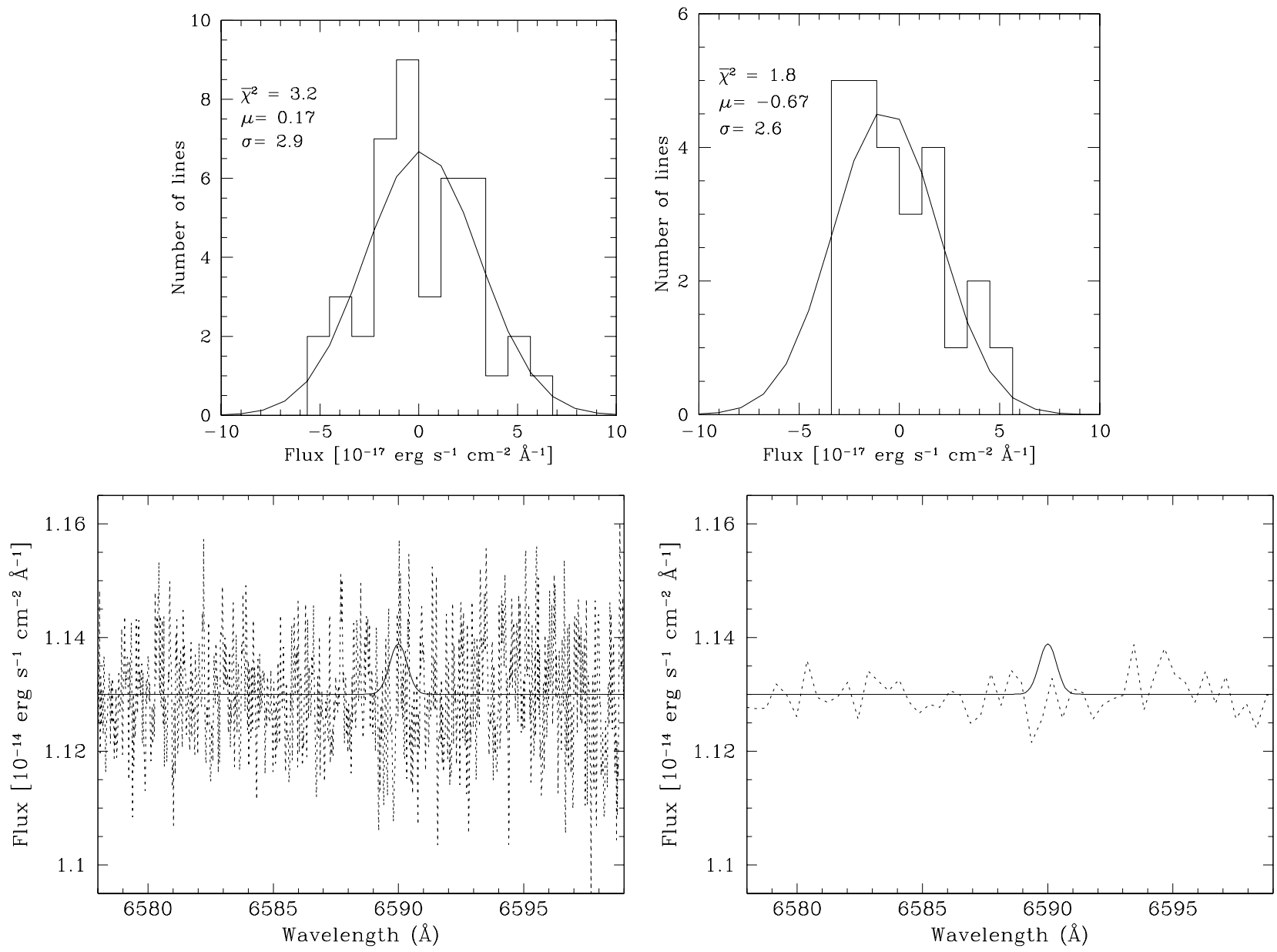

Fig. 5. Histograms of the line profile peak flux density distributions in the the first epoch UVES spectrum at the wavelength range $( \pm 2 \times$ $190 \mathrm{~km} \mathrm{~s}^{-1}$ ) around the expected position of $\mathrm{H} \alpha$ and assuming a line profile with FWHM of $37 \mathrm{~km} \mathrm{~s}^{-1}$ (upper-left) and $62 \mathrm{~km} \mathrm{~s}{ }^{-1}$ (upperright). The histograms have been fitted with Gaussians yielding reduced $\chi^{2}$ s of 3.2 and 1.8 , respectively. The centers $(\mu)$ and widths $(\sigma)$ of the distributions are given in units of $10^{-17} \mathrm{erg} \mathrm{s}^{-1} \mathrm{~cm}^{-2} \AA^{-1}$. Lower-left: the unbinned spectrum $\left(0.77 \mathrm{~km} \mathrm{~s}^{-1}\right.$ pixel $\left.{ }^{-1}\right)$ showing the wavelength region around the expected position of $\mathrm{H} \alpha$ in our first epoch spectrum. A Gaussian line profile with $F W H M$ of $37 \mathrm{~km} \mathrm{~s}^{-1}$ and peak equal to the $3 \sigma$ level is also shown. Lower-right: the same as on the left except that now the spectrum has been rebinned to $\sim 18 \mathrm{~km} \mathrm{~s}^{-1}$ pixel $^{-1}$ resolution. 
Table 2. Comparison of limiting fluxes from two different methods. The center of the Gaussian noise distribution, $\mu$, and its width, $\sigma$, yield $3 \sigma$ upper limit for the emission line profile peak flux density, $f(3 \sigma)_{\mathrm{I}}$. These can be compared to the $f(3 \sigma)_{\text {II }}$ values derived simply from the measured pixel-to-pixel noise in adequately rebinned spectra. The parameters of the flux density distributions $(\mu$ and $\sigma$ ) and the upper limits are given in units of $10^{-17} \mathrm{erg} \mathrm{s}^{-1} \mathrm{~cm}^{-2} \AA^{-1}$.

\begin{tabular}{|c|c|c|c|c|c|c|}
\hline Line & Epoch & $\begin{array}{l}F W H M \\
\left(\mathrm{~km} \mathrm{~s}^{-1}\right)\end{array}$ & $\mu$ & $\begin{array}{l}\sigma \\
17 \mathrm{erg}\end{array}$ & $\begin{array}{l}f(3 \sigma)_{\mathrm{I}} \\
\mathrm{s}^{-1} \mathrm{~cm}^{-2}\end{array}$ & $\begin{array}{l} \\
\quad f(3 \sigma)_{\mathrm{II}} \\
\left.2 \AA^{-1}\right)\end{array}$ \\
\hline $\mathrm{H} \alpha \lambda 6563^{a}$ & 1 & 37 & 0.17 & 2.9 & 8.9 & 11 \\
\hline $\mathrm{H} \alpha \lambda 6563$ & 1 & 62 & -0.67 & 2.6 & 7.1 & 8.1 \\
\hline $\mathrm{H} \alpha \lambda 6563$ & 2 & 37 & 1.1 & 3.6 & 12 & 11 \\
\hline Н $\alpha \lambda 6563$ & 2 & 62 & 1.2 & 2.4 & 8.4 & 8.8 \\
\hline $\mathrm{He} \mathrm{I} \lambda 5876^{b}$ & 1 & 21 & 0.34 & 7.7 & 23 & 29 \\
\hline He I $\lambda 5876$ & 1 & 53 & 1.7 & 5.4 & 18 & 15 \\
\hline He II $\lambda 4686^{c}$ & 1 & 21 & 0.06 & 3.9 & 12 & 14 \\
\hline He II $\lambda 4686$ & 1 & 53 & 0.07 & 2.2 & 6.7 & 7.2 \\
\hline He II $\lambda 4686$ & 2 & 21 & 1.3 & 7.7 & 24 & 23 \\
\hline He II $\lambda 4686$ & 2 & 53 & 1.2 & 5.0 & 16 & 16 \\
\hline
\end{tabular}

a The $\mathrm{H} \alpha$ upper limits were derived for the wavelength range 6580-6597 А.

$b$ The He I upper limits were derived for the wavelength range 5891-5910 ̊̊.

${ }^{c}$ The He II upper limits were derived for the wavelength range 4695-4713 ̊..

$\left(\Delta m_{15}(\mathrm{~B})=1.1\right) \mathrm{SNe}$ Ia (Riess et al. 1999). Mass loss rate and luminosity are close to linearly proportional to each other in the logarithmic space (see Lundqvist et al. 2006 for details). Therefore the uncertainties in the rise time, distance and extinction have only a small effect in the derived mass loss rates. Assuming a wind velocity of $10 \mathrm{~km} \mathrm{~s}^{-1}$ our observed line luminosity limits therefore correspond to mass loss rates of about $<8.9 \times 10^{-6} M_{\odot} \mathrm{yr}^{-1}$ and $<1.2 \times 10^{-5} M_{\odot} \mathrm{yr}^{-1}$, respectively. Somewhat higher upper limits of $\lesssim 1.5 \times 10^{-5} M_{\odot} \mathrm{yr}^{-1}$ have previously been obtained for SNe 1994D (Cumming et al. 1996) and 2000cx (Lundqvist et al. 2006) also assuming $V_{\text {wind }}=$ $10 \mathrm{~km} \mathrm{~s}^{-1}$. However, if $V_{\text {wind }}=50 \mathrm{~km} \mathrm{~s}^{-1}$ is assumed instead, the upper limits increase to about $<4.9 \times 10^{-5} M_{\odot} \mathrm{yr}^{-1}$ and $<6.9 \times 10^{-5} M_{\odot} \mathrm{yr}^{-1}$ for the two epochs, respectively. The mass loss rates observed for symbiotic stellar systems are in the range between $10^{-8} M_{\odot} \mathrm{yr}^{-1}$ and $5 \times 10^{-5} M_{\odot} \mathrm{yr}^{-1}$ (Seaquist et al. 1993) assuming $V_{\text {wind }}=30 \mathrm{~km} \mathrm{~s}^{-1}$. If adopting $V_{\text {wind }}=$ $10 \mathrm{~km} \mathrm{~s}^{-1}$ or $50 \mathrm{~km} \mathrm{~s}^{-1}$ instead, the highest mass loss rates observed for so called Mira type systems with a red giant secondary star would be $\sim 2 \times 10^{-5} M_{\odot} \mathrm{yr}^{-1}$ or $\sim 8 \times 10^{-5} M_{\odot} \mathrm{yr}^{-1}$, respectively. Therefore, our results are not consistent with a symbiotic stellar system in the upper mass loss rate regime being the progenitor of SN 2001el. (See also Sect. 6.)

For He I and He II the $3 \sigma$ line luminosity upper limits are $L_{\mathrm{He}} \lesssim 7 \times 10^{36} \mathrm{erg} \mathrm{s}^{-1}$ and $\lesssim 3 \times 10^{36} \mathrm{erg} \mathrm{s}^{-1}$, respectively, for the first epoch observation assuming a $10 \mathrm{~km} \mathrm{~s}^{-1}$ wind. These are well above the line luminosities predicted by the photoionisation models of Lundqvist et al. (2006). However, future observations covering the $\mathrm{He}$ I $\lambda 10830$ might give more meaningful results for a helium rich wind.
Table 3. $3 \sigma$ upper limits for circumstellar emission line fluxes (in units of $10^{-17} \mathrm{erg} \mathrm{s}^{-1} \mathrm{~cm}^{-2}$ ) assuming an average temperature of $T=$ $28000 \mathrm{~K}$ (see Lundqvist et al. 2006 for details). The upper limits are shown for the wind velocities ranging between $10 \mathrm{~km} \mathrm{~s}^{-1}$ and $50 \mathrm{~km} \mathrm{~s}^{-1}$, i.e., the resulting line widths of $F W H M=37-62 \mathrm{~km} \mathrm{~s}^{-1}$ and $21-53 \mathrm{~km} \mathrm{~s}^{-1}$ for hydrogen and helium lines, respectively. For the first epoch observation the $\mathrm{H} \alpha$ upper limit is also given for the wind velocities of $100 \mathrm{~km} \mathrm{~s}^{-1}$ and $200 \mathrm{~km} \mathrm{~s}^{-1}$.

\begin{tabular}{|c|c|c|c|c|}
\hline Line & $\begin{array}{l}V_{\text {wind }} \\
\left(\mathrm{km} \mathrm{s}^{-1}\right)\end{array}$ & $\begin{array}{l}F W H M \\
\left(\mathrm{~km} \mathrm{~s}^{-1}\right)\end{array}$ & $\begin{array}{l}\text { Epoch } 1 \\
\left(10^{-17} \text { erg s}\right. \\
\end{array}$ & $\begin{array}{l}\text { Epoch } 2 \\
\left.\mathrm{~s}^{-1} \mathrm{~cm}^{-2}\right)\end{array}$ \\
\hline $\mathrm{H} \alpha \lambda 6563$ & 10 & 37 & $<7.7$ & $<10$ \\
\hline Н $\alpha \lambda 6563$ & 50 & 62 & $<10$ & $<12$ \\
\hline $\mathrm{H} \alpha \lambda 6563$ & 100 & 106 & $<15^{a}$ & - \\
\hline $\mathrm{H} \alpha \lambda 6563$ & 200 & 203 & $<20^{a}$ & - \\
\hline He I $\lambda 5876$ & 10 & 21 & $<10$ & $-^{b}$ \\
\hline He I $\lambda 5876$ & 50 & 53 & $<20$ & $-{ }^{b}$ \\
\hline He II $\lambda 4686$ & 10 & 21 & $<4.2^{c}$ & $<8.4$ \\
\hline He II $\lambda 4686$ & 50 & 53 & $<5.9^{c}$ & $<14$ \\
\hline
\end{tabular}

${ }^{a}$ The upper limits for $V_{\text {wind }}$ of $100 \mathrm{~km} \mathrm{~s}^{-1}$ and $200 \mathrm{~km} \mathrm{~s}^{-1}$ are based on the pixel to pixel standard deviations in the rebinned $(1$ pixel $=$ FWHM/2) spectra between $6570 \AA$ and $6608 \AA$.

${ }^{b}$ Due to unsuccessful telluric correction the He I upper limits for the 2nd epoch are significantly higher than for the 1st epoch observation and therefore are not meaningful to present here.

${ }^{c}$ The on-source exposure time for the wavelength region around He II $\lambda 4686$ was twice as long as for the regions around the other lines observed in the first epoch since this region was covered by both the 437 and 564 settings of the UVES instrument.

\subsection{Upper limit for the gas lost from the companion due to the $S N$ explosion}

Marietta et al. (2000) presented two-dimensional simulations of the impact of a SN Ia explosion on a companion star. They studied three different scenarios in which they assumed the companion star to be a main-sequence star, a sub-giant or a red giant star. They found that the amount of of hydrogen rich material lost varied between $\sim 0.002 M_{\odot}$ and $\sim 0.5 M_{\odot}$ depending on the companion star. They also found that most of the hydrogen-rich gas received velocities below $\sim 1000 \mathrm{~km} \mathrm{~s}^{-1}$ in all three scenarios. In order to search for traces of hydrogen in the SN Ia ejecta as revealed by late time emission we have modelled nebular spectra using the code originally described in Kozma \& Fransson (1998), and updated as described in Sollerman et al. (2004) and Kozma et al. (2005). The calculations are based on the explosion model W7 (Nomoto et al. 1984; Thielemann et al. 1986) where we have artificially included varying amounts of solar abundance material.

Based on the calculations by Marietta et al. (2000) we fill the inner region, out to $1000 \mathrm{~km} \mathrm{~s}^{-1}$, in the $\mathrm{W} 7$ model with varying amounts of solar abundance material. Four time dependent calculations were performed including 0.01, 0.05, 0.10 , and $0.50 M_{\odot}$ of solar abundance material, (for further details see Lundqvist et al. 2006). As before an extinction of $A_{\mathrm{V}}=0.78$ and a distance of $D=17.9 \mathrm{Mpc}$ were adopted for SN 2001el. Marietta et al. (2000) also studied the asymmetry of the ejecta due to the impact. The asymmetry effects were not 


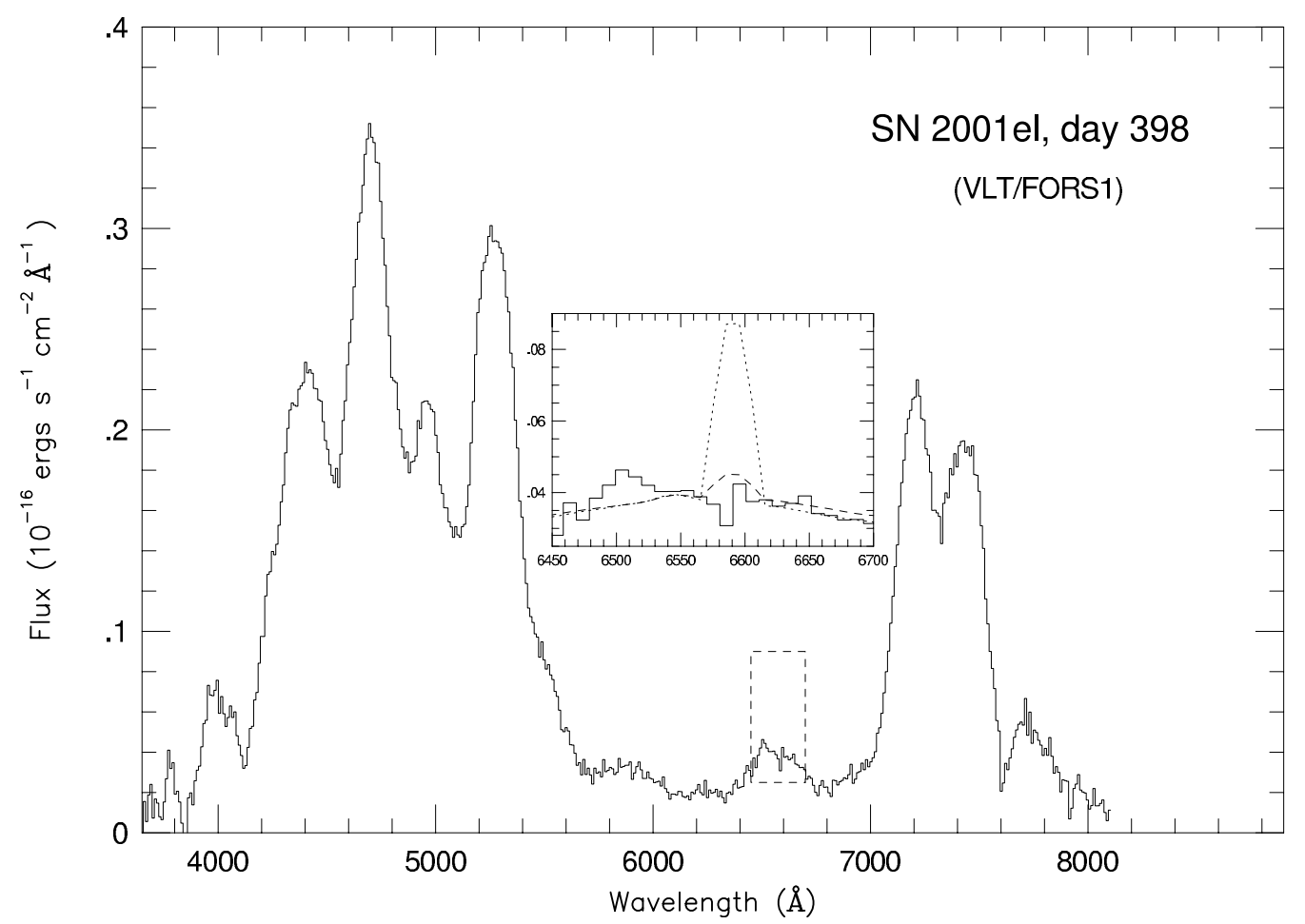

Fig. 6. FORS1 spectrum of SN 2001el obtained on November 2.2 (UT) 2002, i.e., 398 days past the $B$-band maximum light. The inset shows an enlargement of the region marked by dashed lines, focusing on wavelengths around $\mathrm{H} \alpha$ for the redshift of the SN. The SN emission at these wavelengths is dominated by a blend of Fe II emission lines (e.g., $6561 \AA$ ). The dashed and dotted spectra in the inset are models calculated at 380 days for the $\mathrm{SN}$, assuming $0.01 M_{\odot}$ and $0.05 M_{\odot}$ of solar abundance material, respectively, concentrated to $\pm 1000 \mathrm{~km} \mathrm{~s}^{-1}$ from the rest velocity of the supernova. This is to simulate $\mathrm{H} \alpha$ emission from gas removed from a possible companion star according to the models of Marietta et al. (2000). There is no sign of such $\mathrm{H} \alpha$ emission in SN 2001el at these wavelengths, which places a limit on hydrogen rich material of $\sim 0.03 M_{\odot}$. The model used to calculate the late emission is described in Sect. 4.3.
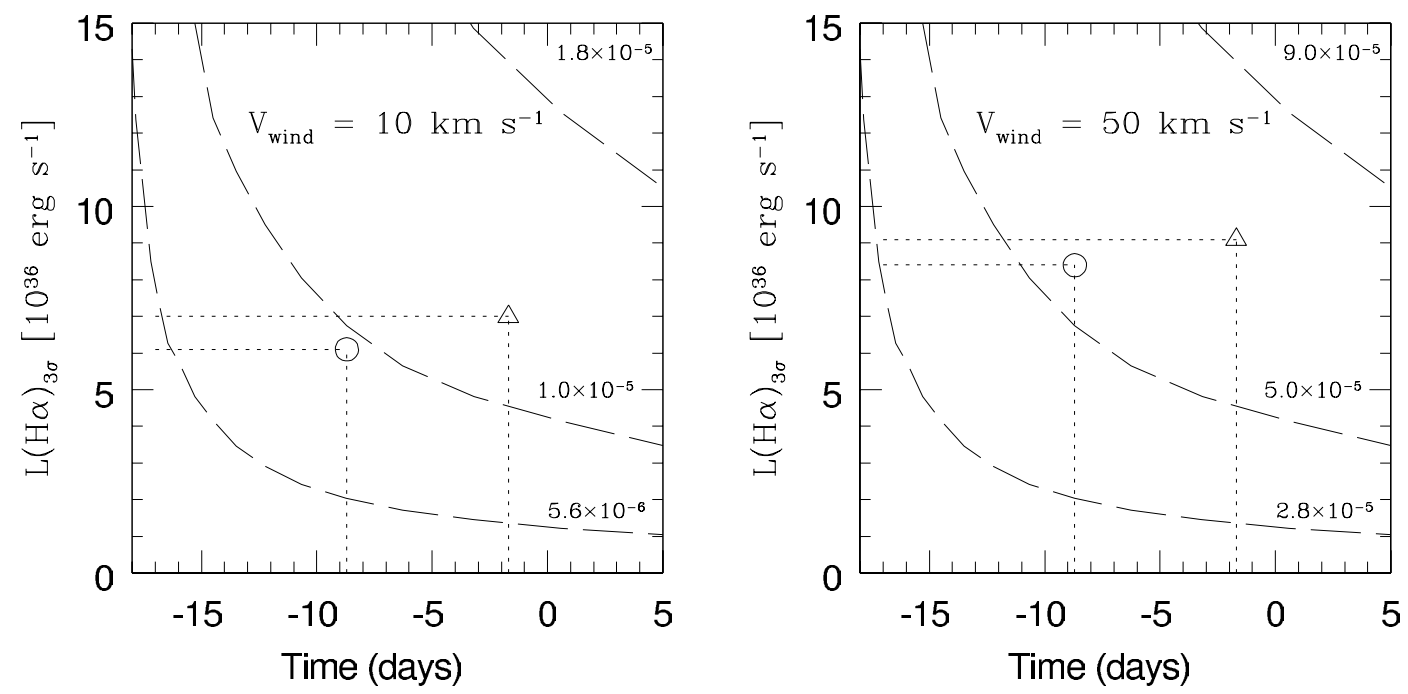

Fig. 7. The modelled evolution of the $\mathrm{H} \alpha$ narrow emission line luminosities are shown for the wind velocity of $10 \mathrm{~km} \mathrm{~s}^{-1}$ (left) and $50 \mathrm{~km} \mathrm{~s}^{-1}$ (right) as a function of the time relative to the $B$-band maximum light (assuming a rise time of 19.5 days for SN 2001el). The three different curves (dashed lines) correspond to mass loss rates of $5.6 \times 10^{-6} M_{\odot} \mathrm{yr}^{-1}, 1.0 \times 10^{-5} M_{\odot} \mathrm{yr}^{-1}$ and $1.8 \times 10^{-5} M_{\odot} \mathrm{yr}^{-1}$, respectively in the LH figure, and $2.8 \times 10^{-5} M_{\odot} \mathrm{yr}^{-1}, 5.0 \times 10^{-5} M_{\odot} \mathrm{yr}^{-1}$ and $9.0 \times 10^{-5} M_{\odot} \mathrm{yr}^{-1}$ in the RH figure. The $3 \sigma$ upper limits for the $\mathrm{H} \alpha$ line luminosity at the two epochs of observation, -8.7 and -1.7 days, are marked with a circle and triangle, respectively.

taken into account in our one-dimensional spherically symmetric models. However, the asymmetries will mainly change the line profiles and will not affect our conclusions on the hydrogen emission.
In Fig. 6 we show, together with the observations of SN 2001el, two modelled spectra at 380 days past explosion containing $0.01 M_{\odot}$ and $0.05 M_{\odot}$ of solar abundance material. We see that even in the model containing $0.05 M_{\odot}$ of 
hydrogen rich material, significant $\mathrm{H} \alpha$ emission is present. This is clearly not seen in the observed spectrum. We therefore conclude that $\sim 0.03 M_{\odot}$ of solar abundance material would have been detected in our late time spectrum via relatively narrow $\left(F W H M \lesssim 1000 \mathrm{~km} \mathrm{~s}^{-1}\right) \mathrm{H} \alpha$ emission. Even such small amounts of solar abundance material show up in the nebular spectrum because the optical depth to gamma-rays in the central, hydrogen rich region is sufficiently high to capture enough gamma-rays to power the radiation from hydrogen. This is in contrast to the $\mathrm{Fe} / \mathrm{Ni}$ rich lower density regions that dominate the emission in the spectrum in Fig. 6 which are predominantly powered by local positron deposition. The mixing of newly synthesised material into the central regions would increase the deposition of positrons in the hydrogen rich material. Therefore, our derived limit on the mass of hydrogen gas from a companion in the central regions of the supernova ejecta should provide a conservative upper limit.

Marietta et al. (2000) found that the amount of stripped and evaporated gas from the companion depends on the nature of the star. The smallest amount of gas lost was for the main-sequence companions at the largest binary separations of six or more stellar radii. They lose $\lesssim 0.02 M_{\odot}$ of solar abundance material due to the impact. However, the mass lost from the main-sequence companions at a smaller binary separation of $~ 3$ stellar radii, subgiant and red giant stars ranges from $0.15 M_{\odot}$ to $\sim 0.5 M_{\odot}$, respectively. Most of this gas has a velocity of $<1000 \mathrm{~km} \mathrm{~s}^{-1}$ as the gas is evaporated from the companion. Scenarios with such high masses are clearly not favoured by our results. We emphasise that the amount of gas stripped off the companion at a high velocity is much lower than the evaporated gas. High-velocity gas may only reveal itself in early spectra (see below).

\section{High-velocity features in SNe 2001el, 1990N, and 1984A}

Early spectroscopic observations of SNe Ia covering wavelengths longer than $\sim 7500 \AA$ are still scarce. Such observations have been reported to show the Ca II IR triplet lines $(\lambda \lambda 8498$, $8542,8662)$ at a high velocity (HV) compared to the SN photospheric velocities for a few SNe Ia. This includes SN 1994D at -8 days (Hatano et al. 1999), SN 1999ee at -7 days (Mazzali et al. 2005a), SN 2000cx at +2 days (Thomas et al. 2004), SN 2001el at -4 days (Wang et al. 2003; Kasen et al. 2003), SN 2003du at -5 days (Gerardy et al. 2004), and SN 2004dt at -7 days (Wang et al. 2004).

Kasen et al. (2003) investigated a plausible geometry for the material giving rise to the observed HV Ca II IR triplet feature in SN 2001el and its spectropolarimetric properties (Wang et al. 2003). They favour a clumpy shell geometrically detached from the photospheric material, and suggest that the HV material could be $\mathrm{Ca}$ rich gas originating in either nuclear burning in the WD or from a swept up accretion disk surrounding the WD.

Thomas et al. (2004) modelled both the HV IR triplet, as well as the HV H\&K feature seen in SN 2000cx at +2 days. They found that $3 \mathrm{D}$ modelling was needed to produce satisfactory spectral fits for both the features. They investigated a scenario where the HV material would be hydrogen or helium rich gas in the SN CSM stripped from a non-degenerate companion star of the progenitor WD as suggested by Marietta et al. (2000; see also Sect. 4.3). The models of Thomas et al. (2004) require at least $\sim 10^{-3} M_{\odot}$ of hydrogen or helium rich material in the HV shell, which they find a factor of ten higher than allowed by the simulations by Marietta et al. (2000) at such a high velocity. Due to the lack of a plausible donor mechanism to the SN CSM, Thomas et al. (2004) conclude that the HV material is more likely to have its origin in the SN explosion itself.

Gerardy et al. (2004) further investigated the scenario of solar abundance material in the SN CSM. They carried out modelling where fast $\mathrm{SN}$ ejecta collide with stationary or slow moving CSM material close to the $\mathrm{SN}$, setting up a forward and reverse shock structure. They assumed that the swept up CSM matter and shocked ejecta matter are mixed in the interaction forming a dense shell of gas with a suitable Ca abundance (solar abundance) to give rise to the observed HV lines. In their modelling three different scenarios produced a shell of $0.02 M_{\odot}$ at 20 days post-explosion which is capable of giving rise to the observed HV Ca II IR triplet feature. We note that the progenitor mass loss rate required by their first scenario is a factor of $\sim 20$ higher than our upper limit for the progenitor of SN 2001el (assuming a $10 \mathrm{~km} \mathrm{~s}^{-1}$ wind). This scenario as well as a scenario with a constant density CSM environment were also found unrealistic by Gerardy et al. (2004) as their energy generation rates would clearly dominate the bolometric light curve of the SN. For SN 2003du Gerardy et al. (2004) favour a scenario where the matter involved in the interaction originates much closer to the progenitor system, in an accretion disk, Roche-lobe, or in a common envelope.

More recently, Mazzali et al. (2005a) found that the observed HV Ca II IR triplet and Si II features in SN 1999ee could be explained by an increase of the mass at the highest ejecta velocities. According to the authors such a density increase could either be due to asymmetries in the explosion, and/or interaction of the ejecta with hydrogen rich material in the CSM of the SN. They found that about $4 \times 10^{-3} M_{\odot}$ of hydrogen rich material in the CSM could explain the HV features observed in SN 1999ee.

\subsection{High-velocity Ca II}

In Fig. 8 we show Ca II profiles of SN 2001el together with the profiles of two other SNe Ia, SN 1984A (Wegner \& McMahan 1987) and SN 1990N (Leibundgut et al. 1991) both showing very high velocities at a similar early epoch. For these figures the spectra were normalised by a first order polynomial fit around the profile of interest, and converted to the velocities w.r.t. to the rest wavelengths assuming $V_{\text {rec }}=1180 \mathrm{~km} \mathrm{~s}^{-1}$, $1010 \mathrm{~km} \mathrm{~s}^{-1}$ and $-261 \mathrm{~km} \mathrm{~s}^{-1}$, for SNe 2001el, 1990N, and 1984A, respectively. To illustrate the maximum extent of the absorbing gas (in velocity space) we used the rest wavelengths of the bluest components of the Ca II triplet/doublet lines viz. $8498 \AA$ and $3934 \AA$ A. However, due to limited wavelength coverage in the $\mathrm{SN} 1990 \mathrm{~N}$ spectrum the normalisation of its Ca II IR triplet profile is unreliable in the red part. Therefore, in the discussion below we will only make use of the blue part of this 
Table 4. Maximum velocities for SNe 1984A, 1990N, and 2001el as indicated by the blue edges of the Ca II H\&K and IR triplet and Si II “6150 ̊” profiles (see Fig. 8).

\begin{tabular}{lllll}
\hline \hline SN & $\begin{array}{l}\text { Epoch } \\
(\text { days })\end{array}$ & $\begin{array}{l}V_{\text {Ca II,H\&K }} \\
\left(\mathrm{km} \mathrm{s}^{-1}\right)\end{array}$ & $\begin{array}{l}V_{\text {Ca II,IR }} \\
\left(\mathrm{km} \mathrm{s}^{-1}\right)\end{array}$ & $\begin{array}{l}V_{\text {Si II }} \\
\left(\mathrm{km} \mathrm{s}^{-1}\right)\end{array}$ \\
\hline $1984 \mathrm{~A}$ & $-7^{a}$ & 38000 & - & 26000 \\
$1990 \mathrm{~N}$ & $-14^{b}$ & 36000 & $\sim 28000$ & 26000 \\
$2001 \mathrm{el}$ & -9 & 34000 & 28000 & 23000 \\
\hline
\end{tabular}

${ }^{a}$ Date of observation 1984 Jan. 10 (Wegner \& McMahan (1987). The epoch adopting Jan. 17 for the $B$-band maximum light (Barbon et al. 1989).

${ }^{b}$ Date of observation 1990 June 26.2 UT (Leibundgut et al. 1991).

profile. In Table 4, we list the maximum velocities as indicated by the blue edges of the normalised HV profiles.

Our first epoch ( -9 days) observation of SN 2001el constitutes to our knowledge the earliest reported coverage $\mathrm{e}^{2}$ of the HV Ca II IR triplet in a SN Ia (in addition to SN 1990N). Our -9 day observation covers also the Ca II H\&K lines enabling comparisons between the velocity profiles of these two features. In Fig. 8a we show profiles of the HV Ca II triplet and doublet lines as observed at -9 days. We illustrate the spectral evolution of the Ca II IR triplet between -9 and -4 days in Fig. $8 \mathrm{~b}$ using the low-resolution spectrum obtained by Wang et al. (2003) for the latter epoch. At both epochs, the IR triplet profile of SN 2001el shows a double dipped structure whereas the H\&K doublet $(3934,3968 \AA)$ feature $\left(\sim 2600 \mathrm{~km} \mathrm{~s}^{-1}\right.$ separation) does not show evidence for two components. The bluemost minimum in the IR triplet profile has been identified to be due to the blend of the $\lambda 8542$ and $\lambda 8498$ components ( $\sim 1500 \mathrm{~km} \mathrm{~s}^{-1}$ separation) whereas the redmost minimum is produced by the $\lambda 8662$ component (Kasen et al. 2003).

The velocities of the centers of the two Ca II profiles at -9 days are quite similar. However, the maximum velocity, as indicated by the blue edge of the profile, is higher for the Ca II H\&K lines $\left(\sim 34000 \mathrm{~km} \mathrm{~s}^{-1}\right)$ than for the IR triplet $\left(\sim 28000 \mathrm{~km} \mathrm{~s}^{-1}\right)$. Similar behaviour was also shown by SN $1990 \mathrm{~N}$ at -14 days with maximum velocities of $36000 \mathrm{~km} \mathrm{~s}^{-1}$ and $\sim 28000 \mathrm{~km} \mathrm{~s}^{-1}$ for the Ca II H\&K and the IR triplet profiles, respectively. This behaviour can be due to blending of the $\mathrm{Ca}$ II $\mathrm{H} \& \mathrm{~K}$ profile with other lines as already pointed out by Kasen et al. (2003) for SN 2001el, but may also just reflect a higher absorption in the H\&K lines. This can be expected since the $H \& \mathrm{~K}$ lines arise from a lower level which may well have a higher population than the level from which the Ca II IR lines are absorbed. However, estimating the non-LTE level populations and the exact contribution of other lines to the observed profile needs detailed modelling of the $\mathrm{SN}$ spectrum. The highest velocity of the three SNe as measured from the blue edges of their Ca II H\&K profiles is shown by SN 1984A (38 $000 \mathrm{~km} \mathrm{~s}^{-1}$ ) at -7 days (see Table 4).

${ }^{2}$ While this paper was being refereed other studies presenting data at even earlier epochs have appeared (see Mazzali et al. 2005b; Quimby et al. 2005).

\subsection{High-velocity Si II}

In Fig. 8, the Si II "6150 ^”" profile is shown for SNe 2001el, $1990 \mathrm{~N}$, and 1984A. Again, the data were normalised and converted to velocities w.r.t. the bluest component of the Si II profile of $6347 \AA$ (see Table 4). In Fig. 8e, the Si II profile of SN 2001el at -9 days is compared with the Si II profiles of SNe 1984A and $1990 \mathrm{~N}$ at -7 and -14 days, respectively. At these epochs both SNe 2001el and 1990N show a flat-bottomed Si II profile. To our knowledge such flat-bottomed Si II lines have not been seen in any other SNe Ia so far.

The pre-maximum light evolution of the Si II profile is illustrated in Fig. 8f for SNe 2001el and 1990N. For the latter epochs we used spectra presented by Wang et al. (2003) and Leibundgut et al. (1991), respectively. In SN 2001el the flat bottom of the line disappeared by the next earliest epoch of observation, 4 days before the maximum light. The same behaviour is also apparent in SN $1990 \mathrm{~N}$ where the shape of the line has changed by the -7 day epoch. In Fig. 5a of Fransson (1984) such a flat-bottomed profile, and its evolution are presented as a result of calculations assuming that the velocity in the SN ejecta is proportional to its radius as expected soon after the SN shock outbreak. The flat-bottomed line shape is produced by pure scattering coming from a thin region compared to the size of the SN photosphere. As the scattering region widens when the SN "photosphere" receeds the flat-bottomed line shape disappears, becoming an ordinary P-Cygni profile (cf., the change from the $R_{2}=1.2$ case to the $R_{2}=1.5$ case in Fig. 5a of Fransson 1984).

The fact that the Ca II lines extend to considerably higher velocities than the Si II line (see Table 4) explains the less pronounced flat section of the Ca II lines. The lower maximum velocity of the $\mathrm{Si}$ II line compared to the Ca II lines is likely to be a result of the larger population of the atomic levels of the $\mathrm{Ca}$ II lines compared to the excited Si II line, and does not need to indicate different abundance distributions. We note that the flat-bottomed Si II profile in SN 1990N has also been explained by a blend of the Si II line with C II $6580 \AA$ line forming in a carbon-rich HV shell detached from the SN photosphere (Fisher et al. 1996), but see Mazzali (2001). Our explanation for the flat-bottomed Si II profile therefore gives less support to an enhanced HV carbon shell.

\section{Discussion and summary}

We have looked for narrow hydrogen and helium lines in our early high-resolution optical spectra of the normal type Ia supernova SN 2001el. No such lines were detected, and we derived upper limits for the luminosities of $\mathrm{H} \alpha$, He I $\lambda 5876$ and He II 14686 . These luminosities were then compared with the photoionisation models of Lundqvist et al. (2006). From the limits for $\mathrm{H} \alpha$ we conclude that the mass loss rate from the progenitor system of SN 2001el was less than $\lesssim 9 \times$ $10^{-6} M_{\odot} \mathrm{yr}^{-1}$ for a spherically symmetric wind with a velocity of $10 \mathrm{~km} \mathrm{~s}^{-1}$ or less than $\$ 5 \times 10^{-5} M_{\odot} \mathrm{yr}^{-1}$ for a velocity of $50 \mathrm{~km} \mathrm{~s}^{-1}$. These estimates are only sensitive to gas outside a few $\times 10^{15} \mathrm{~cm}$ from the $\mathrm{SN}$ explosion site. The gas inside this radius would have been swept up by the SN ejecta 

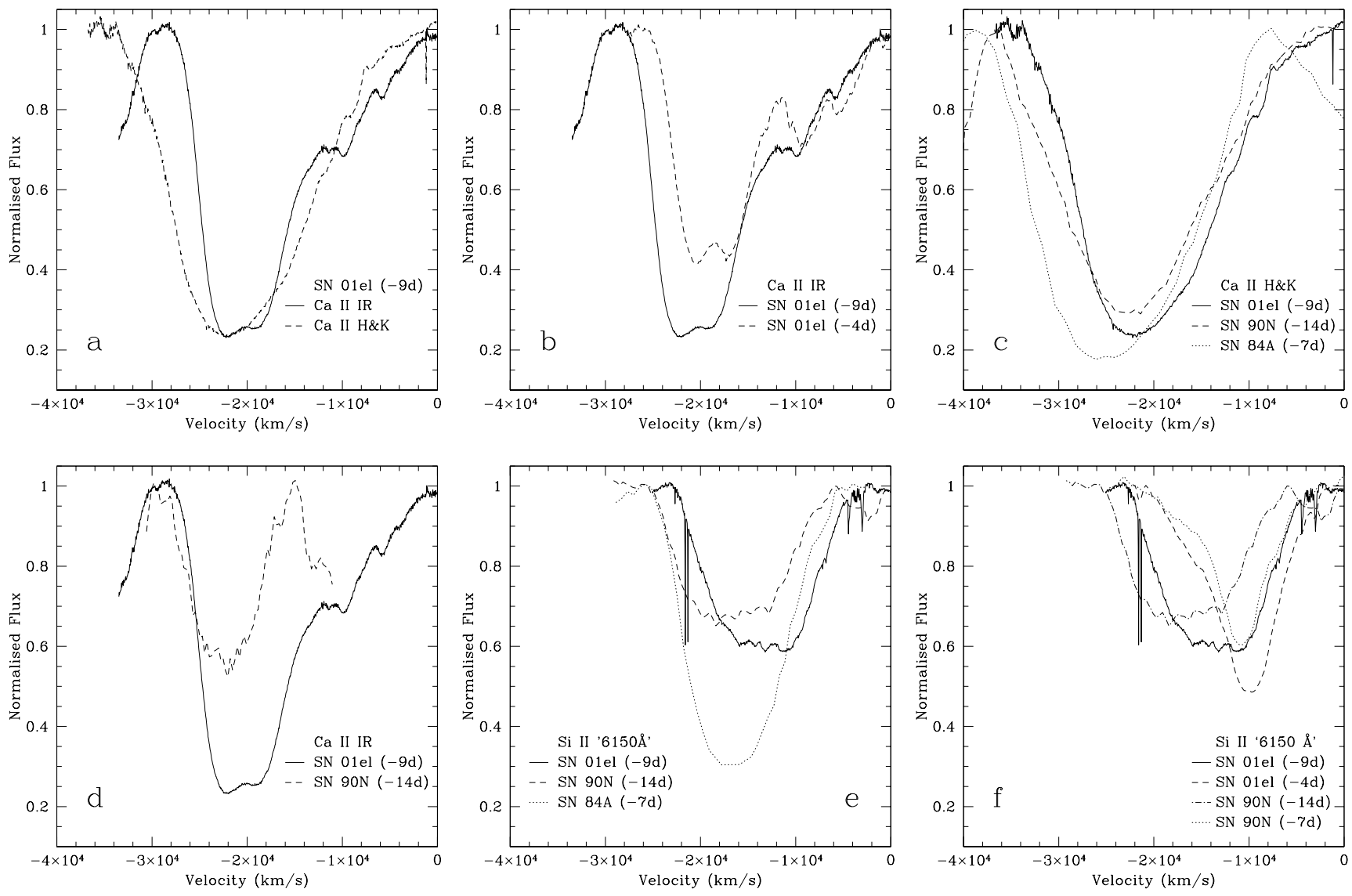

Fig. 8. a) Comparison between the Ca II IR triplet (solid line) and the Ca II H\&K velocity profiles (dashed line) at -9 days. b) Spectral evolution of the Ca II IR triplet from -9 days (solid line) to -4 days (dashed line). c) The Ca II H\&K profiles in SNe 2001el at -9 days (solid line), 1984A at -7 days (dotted line), and SN 1990N at -14 days (dashed line). d) Comparison between the Ca II IR triplet profiles of SNe 2001el (solid line) and 1990N (dashed line). Note that due to the limited wavelength coverage in SN 1990N spectrum the normalisation of its Ca II IR profile is unreliable in the red part. e) Comparisons between the Si II "6150 ̊”" profiles in SNe 2001el at -9 days (solid line), 1984A at -7 days (dotted line), and SN 1990N at -14 days (dashed line). f) Spectral evolution of the Si II "6150 ^’" profile in SNe 2001el (solid and dashed lines) and $1990 \mathrm{~N}$ (dotted and dotted-dashed lines). The flux calibrated $\left(f_{\lambda}\right)$ spectral features have been normalised and converted to velocity space w.r.t. to the rest wavelengths assuming $V_{\text {rec }}=1180,1010$, and $-261 \mathrm{~km} \mathrm{~s}^{-1}$ for SNe 2001el, 1990N, and 1984A, respectively. For this we used the rest wavelengths of the bluest components of the Ca II triplet/doublet lines viz. $8498 \AA$ and $3934 \AA$, and the Si II "6150 ̊" profile of $6347 \AA$.

prior to our observations. However, these are the best $\mathrm{H} \alpha$ based upper limits obtained for a SN Ia so far. Our results, together with the previous observational work on SN 1994D (Cumming et al. 1996) and SN 2000cx (Lundqvist et al. 2006) do not favour a symbiotic star in the upper mass loss rate regime (so called Mira type systems) from being the likely progenitor scenario for these SNe. This is in accordance with the models of Hachisu et al. (1999a,b), which do not indicate a mass loss rate of the binary companion in the symbiotic scenario higher than $\sim 10^{-6} M_{\odot} \mathrm{yr}^{-1}$. Stronger mass loss would initiate a powerful (although dilute) wind from the white dwarf that would clear the surroundings of the white dwarf, and that may even strip off some of the envelope of the companion. The white dwarf wind with its possible stripping effect, in combination with the orbital motion of the stars, is likely to create an asymmetric CSM. Effects of a more disk-like structure of the denser parts of the CSM, and how this connects to our spherically symmetric models, are discussed by Cumming et al. (1996). In general, lower values of $\dot{M} / V_{\text {wind }}$ should be possible to trace in an asymmetric scenario, although uncertainties due the inclination angle and the flatness of the dense part of the CSM would also be introduced. However, to push the upper limits down to the $\sim 10^{-6} \quad M_{\odot} \mathrm{yr}^{-1}$ level a much more nearby ( $D \sim 3 \mathrm{Mpc}$ ) SN Ia needs to be observed even earlier ( $\sim 15$ days before the maximum light) than our first epoch observation of SN 2001el (Mattila et al. in prep.). This will only be feasible by making use of target-of-opportunity (ToO) time offered by observatories such as ESO and Subaru. Similar, or even lower limits on $\dot{M} / V_{\text {wind }}$ can be obtained from radio and X-ray observations (e.g., Eck et al. 2002; Lundqvist et al. 2006), but such observations cannot disentangle the elemental composition of the gas in the same direct way as optical observations can do.

SN 2001el was revisited using VLT/FORS in the nebular phase, $\sim 400$ days after the maximum light. We modelled the late time spectrum to derive an upper limit of $\sim 0.03 M_{\odot}$ for solar abundance material present at velocities lower than $1000 \mathrm{~km} \mathrm{~s}^{-1}$ within the supernova explosion site. This is similar to our results for SNe 1998u and 2000cx (Lundqvist et al. 2006). Comparing this limit to the numerical simulations of Marietta et al. (2000) indicates that symbiotic systems with a 
subgiant, red giant or a main-sequence secondary star at a small binary separation are not likely progenitor scenarios for these $\mathrm{SNe}$ (see also below). These results demonstrate that the combination of very early and late time spectroscopy can be a powerful tool to probe SN Ia progenitors.

Our -9 day spectrum shows that both the Ca II IR triplet and the $\mathrm{H} \& \mathrm{~K}$ lines are present at very high velocities of up to $\sim 28000 \mathrm{~km} \mathrm{~s}^{-1}$ and $\sim 34000 \mathrm{~km} \mathrm{~s}^{-1}$, respectively. Highvelocity Ca II in SNe Ia is, however, not unique for SN 2001el. Here we have highlighted this for SNe 1984A and 1990N, which actually showed $\sim 2000-4000 \mathrm{~km} \mathrm{~s}^{-1}$ higher velocities for the Ca II H\&K as well as the Si II lines at a similar epoch. As a matter of fact, high-velocity $\mathrm{Ca}$ II has now been seen in all SNe Ia (Mazzali et al. 2005b) which have been studied early enough. The presence of the lines confirm the assumption in Lundqvist et al. (2006) that at least some SNe Ia launch their outermost ejecta at velocities in excess of $c / 10$. This means that they sweep up any CSM at a much faster rate than was assumed in the earlier models of Cumming et al. (1996) (see Sect. 4.2).

A fundamental question is of course the origin of the HV Ca II H\&K lines. One possibility mentioned in Sect. 5 is that they form in material stripped off from a non-degenerate companion. From our FORS observation at 400 days postmaximum we were able to put an upper limit of $\sim 0.03 M_{\odot}$ for solar abundance material with a low velocity $\left(\sim 1000 \mathrm{~km} \mathrm{~s}^{-1}\right)$ evaporated from the companion star. According to the models of Marietta et al. (2000) only a small fraction of the gas lost from the companion, $<1 / 1000$, can have a high velocity of $>15000 \mathrm{~km} \mathrm{~s}^{-1}$. The mass of the solar abundance HV gas (originating from the companion star) available to give rise to the HV Ca II lines would therefore be less than $\sim 3 \times 10^{-5} M_{\odot}$ which is a factor of $\sim 3000$ less than the mass of solar abundance gas estimated by Wang et al. (2003) to be required to produce the HV Ca II IR triplet feature seen in SN 2001el. Our observations therefore do not support that the HV Ca II lines would arise in gas stripped from the companion star, which is consistent with the findings of Thomas et al. (2004) for SN 2000cx.

We also note that there is no $\mathrm{H} \beta$ absorption apparent in our spectrum (see Fig. 1) at the velocity of the HV Ca II features. A very tentative identification of such a $\mathrm{HV} \mathrm{H} \beta$ line was made by Branch et al. (2004) in SN 2000cx spectra. The existence of a $\mathrm{HV} \mathrm{H} \beta$ line would have indicated a non-degenerate companion star origin for the HV matter, which is thus not supported by our observations. A very low hydrogen mass upper limit of $\sim 3 \times 10^{-4} M_{\odot}$ was presented by Della Valle et al. (1996) for SN 1990M based on a non-detection of atmospheric $\mathrm{H} \alpha$ absorption in their spectrum near the maximum light. However, this was obtained by using a simplified model. $\mathrm{H} \alpha$ is usually in net emission in supernovae, and thus the absorption is weaker than would be predicted by an analysis using resonant scattering. The emission falls into the Si II absorption trough and so could be hidden in SNe Ia (Thomas et al. 2004; Branch et al. 2004). This effect is somewhat less for $\mathrm{H} \beta$ and $\mathrm{H} \gamma$. Lentz et al. (2002) performed detailed spectral synthesis calculations of mixed solar material into a W7 model and found that even relatively large amounts of hydrogen $\left(\sim 10^{-3} M_{\odot}\right)$ would be hard to detect at the earliest times. Furthermore, early time observations can only detect hydrogen present in the outer parts of the supernova ejecta, or in a detached wind (see Sect. 4.2). However, as the simulations of Marietta et al. (2000) predict only a very small high-velocity tail for the hydrogen rich material, early observations seem futile to reveal any stripped material from the companion. The models of Marietta et al. (2000), in combination with our results in Sect. 4.3 and in Lundqvist et al. (2006), show that stripped, or rather evaporated material from a companion, should instead be looked for at late times. Our limits on the late emission are actually so tight that most of the scenarios in Marietta et al. (2000) appear to be ruled out. This, in combination with the properties of the presumed binary companion of the Tycho progenitor (Ruiz-Lapuente et al. 2004), should be used to find a viable solution to the singledegenerate progenitor scenario.

Another possibility for the HV Ca II lines mentioned in Sect. 5 is that they arise in gas compressed due to circumstellar interaction (Gerardy et al. 2004). The scenario with a dense continuous wind outside a few $\times 10^{15} \mathrm{~cm}$ is not supported by our observations and models of narrow circumstellar emission lines. Furthermore, the lack of interaction signatures in the bolometric light curves (e.g. Gerardy el al. 2004) and the lack of radio emission in normal SNe Ia (e.g., Eck et al. 2002), do not support such a scenario either. The model with a circumstellar disk could be more likely, although we note that such a scenario should produce a range of velocities for the $\mathrm{Ca}$ II absorption depending on the viewing angle, unless the disk is thick. More self-consistent modelling of the circumstellar interaction is needed to see whether a circumstellar disk model can explain the HV Ca II features seen in SNe Ia.

A more natural explanation for the HV Ca II lines and the HV Si II lines in SN 2001el and other SNe Ia would appear to be absorption in the outermost parts of the SN ejecta which extend to high enough velocities. We discussed this qualitatively in Sect. 5.2. Maximum velocities of up to $\sim 26000 \mathrm{~km} \mathrm{~s}^{-1}$ and $\sim 38000 \mathrm{~km} \mathrm{~s}^{-1}$ were observed for the blue edges of the Si II and $\mathrm{Ca}$ II H\&K profiles, respectively, in the -7 day spectrum of SN 1984A. Lentz et al. (2001) modelled the early spectra of SN 1984A and found that the large blueshifts could be produced by a higher density in the outer layers of the ejecta. Furthermore, Hatano et al. (2000) showed that even at 10 days past maximum light, i.e., much later than the times studied here, there exists a class of "fast" supernovae typified by SN 1984A. More recently Benetti et al. (2005) divided "normal" SNe Ia into groups showing high (HVG) and low (LVG) temporal velocity gradients for the Si II profile after the maximum light. Both SNe 2001el and 1990N belong to the LVG group whereas SN 1984A belongs to the HVG SNe which also tend to show the highest photospheric velocities both before and after the maximum light. It is important to understand whether the density structure variations of the HV SNe Ia are due to initial conditions, variations in the explosion mechanism or something else. How these HV SNe Ia are correlated with brightness at maximum light is therefore an important question for the use of SNe Ia as cosmological probes.

Acknowledgements. The VLT/UVES observations were obtained in ToO service mode. We thank the Paranal staff for their help with the observations, and Robert Cumming for helpful discussions and 
comments on the manuscript. We thank Lifan Wang for letting us to use his spectrum of SN 2001el from the epoch -4 days and Stefano Benetti for the early spectra of SN 2002bo. S.M. acknowledges financial support from the "The Physics of Type Ia SNe" Research Training Network under contract HPRN-CT-2002-00303. This work was also supported by the Swedish Research Council, the Swedish Space Board, and the Royal Swedish Academy of Sciences. P.L. is a Research Fellow at the Royal Swedish Academy supported by a grant from the Wallenberg Foundation. E.B. was supported in part by grants from NASA and the NSF. K.N. was supported by the Japan Society for the Promotion of Science.

\section{References}

Aldering, G., Humphreys, R. M., \& Richmond, M. 1994, AJ, 107, 662 Ballester, P., Modigliani, A., Boitquin, O., et al. 2000, ESO Messenger, 101, 31

Ballester P., Boitquin O., Modigliani, A., \& Wolf, S. 2003, UVES Pipeline (v.2.0) User's Manual

Barbon, R., Rosino, L., \& Iijima, T. 1989, A\&A, 220, 83

Baron, E. 2003, Nature, 424, 628

Benetti, S., Meikle, P., Stehle, M., et al. 2004, MNRAS, 348, 261

Benetti, S., Cappellaro, E., Mazzali, P. A., et al. 2005, ApJ, 623, 1011

Branch, D., Thomas, R. C., Baron, E., et al. 2004, ApJ, 606, 413

Chugai, N. N., Chevalier, R. A., \& Lundqvist, P. 2004, MNRAS, 355 , 627

Chevalier, R. A. 1982, ApJ, 258, 790

Cumming, R. J., Lundqvist, P., Smith, et al. 1996, MNRAS, 283, 1355

Della Valle, M., Benetti, S., \& Panagia, N. 1996, ApJ, 459, L23

Deng, J., Kawabata, K. S., Ohyama, Y., et al. 2004, ApJ, 605, L37

de Vaucouleurs, G., de Vaucouleurs, A., Corwin, H. G., et al. 1991, Third Reference Catalogue of Bright Galaxies (Berlin, Heidelberg, New York: Springer-Verlag)

Eck, C. R., Cowan, J. J., \& Branch, D. 2002, ApJ, 573, 306

Eldridge, J. J., \& Tout, C. A. 2004, MNRAS, 353, 87

Fransson, C. 1984, A\&A, 132, 115

Fryxell, B. A., \& Arnett, W. D. 1981, ApJ, 243, 994

Fisher, A., Branch, D., Nugent, P., \& Baron, E. 1997, ApJ, 481, L89

Gerardy, C. L., Hoflich, P., Fesen, R. A., et al. 2004, ApJ, 607, 391

Hachisu, I., Kato, M., \& Nomoto, K. 1999a, ApJ, 522, 487

Hachisu, I., Kato, M., Nomoto, K., \& Umeda, H. 1999b, ApJ, 519, 314

Hamuy, M., Suntzeff, N. B., Heathcote, S. R., et al. 1994, PASP, 106, 566

Hamuy, M., Phillips, M. M., Suntzeff, N. B., et al. 2003, Nature, 424, 651

Hatano, K., Branch, D., Fisher, A., et al. 1999, ApJ, 525, 881

Hatano, K., Branch, D., Lentz, E. J., et al. 2000, ApJ, 543, L49

Hillebrandt, W., \& Niemeyer, J. C. 2000, ARAA, 38, 191

Iben, I. Jr., \& Renzini, A. 1983, Annual Rev. Astron. Astrophys., 21, 271

Kasen, D., Nugent, P., Wang, L., et al. 2003, ApJ, 593, 788

Kotak, R., Meikle, W. P. S., Adamson, A., \& Leggett, S. K. 2004, MNRAS, 354, L13

Kozma, C., \& Fransson, C. 1998, ApJ, 496, 946

Kozma, C., Fransson, C., Hillebrandt, W., et al. 2005, A\&A, 437, 983
Krisciunas, K., Suntzeff, N. B., Candida, P., et al. 2003, AJ, 125, 166

Leibundgut, B., Kirshner, R. P., Filippenko, A. V., et al. 1991, ApJ, 371, L23

Lentz, E. J., Baron, E., Branch, D., \& Hauschildt, P. H. 2001, ApJ, 547,402

Lentz, E. J., Baron, E., Hauschildt, P. H., \& Branch, D. 2002, ApJ, 580,374

Livne, E., Tuchman, Y., \& Wheeler, J. C. 1992, ApJ, 399, 665

Lundqvist, P., Sollerman, J., Leibundgut, B., et al. 2003, From Twilight to Highlight: The Physics of Supernovae, ed. W. Hillebrandt, \& B. Leibundgut, 309

Lundqvist, P., Mattila S., Sollerman, et al. 2005, in the CD version of the Proceedings Supernovae, ed. J. M. Marcaide, \& K. W. Weiler, IAU Coll., 192, 81 [arXiv: astro-ph/0309006]

Lundqvist, P., et al. 2006, A\&A, to be submitted http://www. astro.su.se/ peter/uves_papers/

Marietta, E., Burrows, A., \& Fryxell, B. 2000, ApJS, 128, 615

Mathewson, D. S., \& Ford, V. L. 1996, ApJS, 107, 97

Maund, J. R., Smartt, S. J., Kudritzki, et al. 2004, Nature, 427, 129

Mazzali, P. 2001, MNRAS, 321, 341

Mazzali, P. A., Benetti, S., Stehle, M., et al. 2005a, MNRAS, 357, 200

Mazzali, P. A., Benetti, S., Altavilla, G., et al. 2005b, ApJ, 623, L37

Meikle, W. P. S. 2000, MNRAS, 314, 782

Monard, A. G. 2001, IAUC, 7720

Nomoto, K., Thielemann, F.-K., \& Yokoi, K. 1984, ApJ, 286, 644

Nomoto, K., et al. 2000, in Type Ia Supernovae: Theory and Cosmology, ed. J. Niemeyer, \& J. Truran (Cambridge: Cambridge Univ. Press), 63

Nomoto, K., et al. 2004, in Frontier in Astroparticle Physics and Cosmology, ed. K. Sato, \& S. Nagataki (Tokyo: Universal Academy Press), 323 [arXiv: astro-ph/0406263]

Phillips, M. M., Lira, P., Suntzeff, N. B., et al. 1999, AJ, 118, 1766

Piskunov, N. E., \& Valenti, J. A. 2002, A\&A, 385, 1095

Quimby, R., Höflich, P., Kannappan S. J., et al. 2005, ApJ, in press [arXiv: astro-ph/0509304]

Rieke, G. H., \& Lebofsky, M. J. 1985, ApJ, 288, 618

Riess, A. G., Filippenko, A. V., Li, W., et al. 1999, AJ, 118, 2675

Ruiz-Lapuente, P., Comeron, F., Mendez, J., et al. 2004, Nature, 431, 1069

Seaquist, E. R., Krogulec, M., \& Taylor, A. R. 1993, ApJ, 410, 260

Smartt, S. J., Maund, J. R., Gilmore, et al. 2003, MNRAS, 343, 735

Smartt, S. J., Maund, J. R., Hendry, M. A., et al. 2004, Science, 303, 499

Sollerman, J., Leibundgut, B., \& Lundqvist, P. 2001, IAUC, 7723

Sollerman, J., Lindahl, J., Kozma, C., et al. 2004, A\&A, 428, 555

Sollerman, J., Cox, N., Mattila, S., et al. 2005, A\&A, 429, 559

Taam, R. E., \& Fryxell, B. A. 1984, ApJ, 279, 166

Thielemann, F.-K., Nomoto, K., \& Yokoi, K. 1986, A\&A, 158, 17

Thomas, R. C., Branch, D., Baron, E., et al. 2004, ApJ, 601, 1019

Tinney, C. G., \& Reid, I. N. 1998, MNRAS, 301, 1031

Walborn, N. R., Prevot, M. L., Prevot, L., et al. 1989, A\&A, 219, 229

Wang, L., Baade, D., Höflich, P., et al. 2003, ApJ, 591, 1110

Wang, L., Baade, D., \& Höflich, P. 2005, ApJ, submitted [arXiv: astro-ph/0409593]

Wegner, G., \& McMahan, R. K. 1987, AJ, 93, 287

White, G. L., \& Malin, D. F. 1987, Nature, 327, 36 